\title{
Pneumococcal Pneumonia---Risky Business
}

\author{
Invited Lecture Abstracts
}

ISPPD-0532

Pneumococcal Pneumonia---Risky Business

\section{WHAT NEW DIAGNOSTICS TELL US ABOUT THE ROLE OF PNEUMOCOCCUS IN THE ETIOLOGY OF PNEUMONIA}

\author{
D.R. Murdoch ${ }^{1}$ \\ ${ }^{1}$ New Zealand, University of Otago, Christchurch, New Zealand
}

The importance of Streptococcus pneumoniae as a pneumonia pathogen is beyond question. S. pneumoniae is regarded as the single biggest cause of community-acquired pneumonia across all age groups and geographic regions of the world. However, diagnosing pneumococcal pneumonia in individual patients can be difficult, mainly due to the inability to obtain specimens from the site of infection and the failure of diagnostic tests to distinguish colonization from infection. Regardless of these shortcomings, newer diagnostics have enhanced understanding of the role of $S$. pneumoniae in the etiology of pneumonia in several ways. First, they have reinforced the importance of $S$. pneumoniae as the most commonly identified cause of pneumonia. For example, the latest generation pneumococcal urinary antigen lateral flow tests have improved the ability to diagnose pneumococcal pneumonia in adults. Second, measurement of pneumococcal load provides insights into the pathogenesis of pneumonia and may be helpful in distinguishing colonization from infection. In particular, preliminary data indicates that quantitative PCR testing of upper respiratory tract specimens may have diagnostic utility. Third, newer diagnostics have increased recognition that pneumococcus is often present with other potential pathogens. These observations reinforce the concept that infection with multiple pathogens may be important in the pathogenesis of pneumonia. There is a pressing need for completely new approaches to pneumonia diagnostics, particularly approaches that can distinguish colonising from infecting microorganisms, that can distinguish recent from past infection, that account for host responsiveness, and can utilise samples that are easy to obtain.

No conflict of interest

\section{Oral Plenary Abstracts}

ISPPD-0166

Pneumococcal Pneumonia---Risky Business

\section{PRELIMINARY RESULTS OF THE PILOT PNEUMONIA MULTICENTRIC STUDY IN THE GABRIEL NETWORK FOR ETIOLOGICAL AGENTS IDENTIFICATION}

V. Picot ${ }^{1}$, M. Messaoudi ${ }^{1}$, J.N. Telles ${ }^{1}$, F. Komurian-Pradel ${ }^{1}$, G. Vernet ${ }^{1}$, T.H.E. Pneumonia study members ${ }^{2}$, G. Paranhos-Baccalà ${ }^{1}$

${ }^{1}$ Emerging Pathogens Laboratory, Fondation Mérieux, Lyon, France; ${ }^{2}$ GABRIEL network, Fondation Mérieux, Lyon, France

Background and Aims: The final purpose of the Pilot Multi-centric Pneumonia Study (launched in 2010) is to provide information on the pneumonia etiology (viruses and bacterial) to improve case management of the pneumonia ill child.

Methods: A hospital-based case-control study has been carried-out in 10 centers located in 9 countries over 3 continents. 826 cases and 791 controls out of 2,000 under 5 years of age were enrolled. Respiratory specimens and whole blood were collected from all pneumonia cases and controls to identify viruses or bacteria using real-time multiplex PCR assays. Streptococcus pneumoniae was serotyped in positive samples.

Results: The main bacterial agent found in cases and control respiratory samples were S. pneumoniae (58\% and $40 \%$, respectively). Staphylococcus aureus, Haemophilus influenzae b, Moraxella pneumoniae and Chlamydia pneumoniae were less represented ( $18 \%$ to $0.1 \%$ ). For the viral population, RSV was the principal agent found ( $26 \%$ in cases and only at 5\% in controls). HMPV, PIV-3 and Influenza-A/B were found mainly in cases. Rhinoviruses were found equally in controls and cases (around 21\%). The main S. pneumoniae serotypes represented were 6A/B; 14; $19 \mathrm{~A} ; 19 \mathrm{~F}$ and 5 . The viral and S. pneumoniae serotype populations were different in each country. These results while preliminary are nonetheless interesting.

Conclusions: The objective of this study is to identify the etiologies of pneumonia among hospitalized children in developing and emergent countries. The results will improve individual care and propose appropriate public health policies. The results should permit a better implementation of targeted curative or preventive measures.

No conflict of interest 
ISPPD-0366

Pneumococcal Pneumonia---Risky Business

\section{PREVALENCE OF NEWLY-DISCOVERED RESPIRATORY VIRUSES IN HIV-INFECTED AND HIV-UNINFECTED CHILDREN HOSPITALIZED WITH PNEUMONIA AND PROBING THE ROLE OF PNEUMOCOCCAL CO-INFECTION}

M.C. Nunes ${ }^{1}$, Z. Kuschner ${ }^{2}$, Z. Radebe ${ }^{1}$, C.L. Cutland ${ }^{1}$, R. Madimabe ${ }^{1}$, L. Kuwanda ${ }^{1}$, K.P. Klugman ${ }^{1}$, S.A. Nzenze ${ }^{1}$, P.V. Adrian ${ }^{1}$, S.A. Madhi ${ }^{1}$

${ }^{1}$ Faculty of Health Sciences University of the Witwatersrand, Respiratory and Meningeal Pathogens Unit, Johannesburg, South Africa;

${ }^{2}$ School of Medicine Stony Brook, Stony Brook University, New York, USA

Advances in molecular diagnostics have expanded the repertoire of respiratory viruses implicated in the pathogenesis of pneumonia. We aimed to determine the prevalence of human bocavirus (hBoV), human rhinovirus (hRV), polyomavirus-WU (WUPyV) and -KI (KIPyV) and human coronaviruses (CoV)-OC43, -NL63 and -HKU1 among children hospitalized with lower respiratory tract infections (LRTI) and probe the role of pneumococcal co-infection in HIV-infected and -uninfected children. Multiplex real-time reverse transcriptase-polymerase chain reaction was undertaken on archived nasopharyngeal aspirates obtained from a cohort of 39836 children randomized to receive 9-valent pneumococcal conjugate vaccine (PCV9) or placebo when hospitalized for LRTI. At least one of the newly-studied viruses was identified in 53\% (275/518) and 54\% (509/943) of LRTI-episodes in HIV-infected and -uninfected children, respectively. The newly-studied viruses were detected at high frequency in association with other respiratory viruses (22.8\% in HIV-infected and $28.5 \%$ in HIV-uninfected children). Fully PCV9-vaccinated HIV-uninfected children had a lower incidence of hospitalization for KIPyV $(80.0 \%$; 95\% $\mathrm{Cl}: 41.4,93.2)$, WUPyV (48.5\%; 95\% Cl: $9.1,70.8$ ) and overall coronaviruses $(60.0 \% ; 95 \% \mathrm{Cl}: 9.1,82.4)$. There was, however, no difference in incidence of $\mathrm{hBoV}$ and $\mathrm{hRV}$ between PCV9 and placebo recipients among HIV-uninfected children. Also, no differences in incidence of pneumonia hospitalization were observed between PCV9 and placebo recipients among HIV-infected children. We established a high prevalence of the newly studied viruses in HIV-infected and HIVuninfected children hospitalized for LRTI. Using a PCV-probe approach, we established that pneumonia associated with polyomaviruses and coronaviruses are probably precipitated by pneumococcal co-infection and preventable by PCV immunization.

No conflict of interest

ISPPD-0336

Pneumococcal Pneumonia---Risky Business

\section{CONTINUED DECLINE IN PNEUMONIA HOSPITALIZATIONS IN YOUNG CHILDREN FOLLOWING TRANSITION FROM PCV7 TO PCV13 IN TENNESSEE}

\section{M.R. Griffin ${ }^{1}$, M.R. Moore ${ }^{2}$, C.G. Whitney ${ }^{2}$, C.G. Grijalva ${ }^{1}$}

${ }^{1}$ Health Policy, Vanderbilt University, Nashville, USA; ${ }^{2}$ National Center for Immunization and Respiratory Diseases, Centers for Disease Control and Prevention, Atlanta, USA

Background and Aims: Pneumonia hospitalizations in US children age <2 years declined $43 \%$ from 12.7 before PCV7 introduction (1997-1999) to 7.2 per 1000 after (2007-2009). We assessed whether Tennessee pneumonia trends were similar and whether declines continued following PCV13 introduction.

Methods: Tennessee residents age $<2$ years with pneumonia as a reason for admission were identified 1/98 through 6/12 from Tennessee's Hospital Discharge Data System. We performed a time-series analysis of annualized monthly rates of pneumonia hospitalization and compared the observed annual rate by 2012 to expected rates based on trends from baseline (1/98-12/99) and PCV7 (1/01-6/09) years. PCV7 (2000) and PCV13 (7/09-6/10) introduction and pandemic periods were excluded from analyses. Fractures served as a control condition.

Results: By 2012, the pneumonia hospitalization rate was 4.2 per 1000 Tennessean children <2 years, representing a decline of $23 \%$ (95\% confidence interval $9 \%, 34 \%$ ) from the expected rate of 5.4 based on PCV7 years trend and a decline of $65 \%(59 \%, 69 \%)$ from the expected rate of 11.8 per 1000 based on baseline trend (figure). These declines represented approximately 1212 and 195 fewer pneumonia hospitalizations than expected annually based on expected rates from baseline and PCV7 years, respectively. There was no decline in fractures.

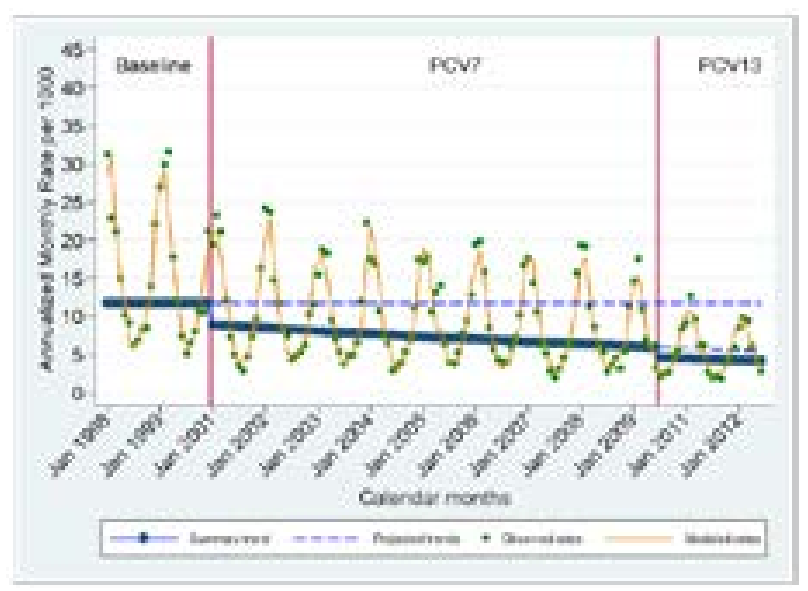


Conclusion: Historically low pneumonia hospitalizations among young children in 2012 demonstrate early benefits from transition from PCV7 to PCV13.

Conflict of interest

ISPPD-0556

Pneumococcal Pneumonia---Risky Business

\title{
IMPACT OF PCV10 INTRODUCTION ON PNEUMONIA MORTALITY RATES IN BRAZIL: A TIME SERIES ANALYSIS
}

\author{
R. Minamisava ${ }^{1}$, S. Sgambatti ${ }^{2}$, O.L. Morais-Neto ${ }^{3}$, E.B. Cristo ${ }^{3}$, J.J.C. Escalante ${ }^{4}$, A.L. Bierrenbach ${ }^{5}$, A.L. Andrade ${ }^{6}$
}

${ }^{1}$ Department of Nursing, School of Nursing, Goiania, Brazil; ${ }^{2}$ Department of Medicine, Pontificial Catholic University, Goiania, Brazil;

${ }^{3}$ Department of Community Health, Institute of Tropical Pathology and Public Health Federal University of Goias, Goiania, Brazil; ${ }^{4} \mathrm{De}-$ partment of Analysis of Health Situation, Ministry of Health Brasilia Brazil, Brasilia, Brazil; ${ }^{5}$ Department of Epidemiology and Research, Research and Education Institute Hospital Sirio-Libanes, Sao Paulo, Brazil; ${ }^{6}$ Department of Community Health, Institute of Tropical Pathology and Public Health - Federal University of Goias, Goiania, Brazil

Background: Community-acquired pneumonia is a leading cause of mortality globally, particularly among infants and the elderly. Brazil introduced the PCV10 in the routine immunization in 2010, targeting children aged 2-23 months. We aimed to assess the impact of PCV10 on pneumonia deaths, three years after vaccination start. Methods: Brazilian Mortality Information System data from 2005 to 2012 were used to conduct an interrupted time-series analysis for patients residing in all 26 State capitals and the Federal District. Pneumonia death (primary cause) was defined by ICD-10 codes J12-J18. All respiratory causes of death (except ICD10 codes J09-J18) were used as the comparator. Monthly denominators were estimated from Census data. Pre-vaccination period (2005-2009, excluding H1N1 pandemic months) was used to ascertain the predicted pneumonia mortality rates post-vaccination (2011-2012) using Holt-Winters method to control for trend and seasonal variations. Monthly-observed mortality rates for the pneumonia and the comparator groups were compared with predicted values in all age-groups, and the percentage changes were calculated, with $95 \% \mathrm{Cls}$ and respective $\mathrm{p}$-values.

Results: Pneumonia mortality rates in children aged $2-23$ months decreased $15.5 \%(95 \% \mathrm{Cl},-38.2 \% ; 7.2 \%, p=0.06)$ in post-vaccination period, while mortality due to respiratory causes increased $1.4 \%(95 \% \mathrm{Cl},-16.7 \% ; 19.5 \%, p=0.92)$; an overall reduction of $16.9 \%$ was estimated when comparing pneumonia vs respiratory causes $(p=0.001)$. No significant decreases in pneumonia mortality were observed in other age-groups.

Conclusion: Our findings point to a decrease in pneumonia mortality rates in infants targeted by the immunization program, 3 years after vaccination start. Herd effect was not yet observed.

No conflict of interest

\section{Oral Poster Abstracts}

\author{
ISPPD-0434
}

Pneumococcal Pneumonia---Risky Business

\section{CHEST RADIOGRAPHY FINDINGS IN PNEUMOCOCCAL PNEUMONIA CASES IN THE PERCH STUDY}

The PERCH Study Group ${ }^{1}$

${ }^{1}$ International Vaccine Access Center, The Johns Hopkins Bloomberg School of Public Health, Baltimore, USA

Background and Aims: Vaccine probe studies have shown that obvious alveolar consolidation on chest x-rays (WHOdefined chest x-ray confirmed pneumonia; CXR-AC), is associated with pneumococcal and Haemophilus influenzae type $b$ pneumonia. In this analysis, we assess the diagnostic accuracy of standardized CXRs in microbiologically confirmed pneumococcal pneumonia (MCPP) cases in the Pneumonia Etiology Research for Child Health (PERCH) study.

Methods: Pneumonia Etiology Research for Child Health (PERCH) is a case-control study of pneumonia etiology among children aged 1-59 months hospitalized with WHO-defined severe or very severe pneumonia in seven African and Asian countries. Standardized biological specimens, testing and a CXR were obtained at admission. CXRs were dual-read by a trained panel of 14 pediatricians and radiologists as CXR-AC, other infiltrate or normal, and an arbitration panel resolved discordant readings. MCPP cases were positive for Streptococcus pneumoniae by culture of blood, lung or pleural aspirate, or were lytA positive in lung or pleural aspirates. Sensitivity/specificity of CXR for MCPP was calculated.

Results: Overall, 526 cases (24.9\%) had CXR-AC (site-specific range, 8.6\% - 41\%). Of 2112 enrolled cases with interpretable CXR findings, 33 had MCPP. Of these, $22(67 \%)$ had CXR-AC, $4(12 \%)$ had other infiltrates only and 7 (21\%) were normal. Of 2079 non-MCPP cases, 504 (24\%) had CXR-AC, 583 (28\%) other infiltrates, and 992 (48\%) were normal. CXR-AC specificity for MCPP was $75.8 \%$, positive predictive value $4.2 \%$, and negative predictive value 99.3\%.

Conclusion: WHO-defined CXR-AC is associated with pneumococcal pneumonia in the PERCH study; however, approximately one-third of pneumococcal pneumonias do not present with primary endpoint pneumonia (PEP).

No conflict of interest 
ISPPD-0098

Pneumococcal Pneumonia---Risky Business

\title{
A 13-VALENT PNEUMOCOCCAL CONJUGATE VACCINE SAVED MORE IN-PATIENT CARE COSTS DUE TO PNEUMONIA IN INFANTS COMPARED WITH A 10-VALENT VACCINE
}

\author{
A. Berglund ${ }^{1}$, M. Ekelund ${ }^{1}$, L. Nyman ${ }^{1}$ \\ ${ }^{1}$ Medical and Access Unit, Pfizer AB, Sollentuna, Sweden
}

Background and Aims: Pneumococcal vaccination of infants is part of a national immunization program in many countries. In most of these, only one of two vaccines is chosen for the entire population, either a vaccine with 10 or a vaccine with 13 serotypes (PCV10 and PCV13), but in Sweden there is a split market. We aimed to address the potential effect of PCV10 and PCV13 on the incidence of in-patient pneumonia but also potential costs savings among vaccinated children.

Methods: The incidence per quarter of in-patient pneumonia cases (ICD-10 J12-J18) was identified for all regions among children $<2$ years between 1998 and 2012. All regions had implemented a program of pneumococcal vaccination by 2009 starting with PCV7. In 2010, some regions switched to PCV10, whereas others switched to PCV13.

Results: Among children $<2$ years and following adjustment for region, seasonal variation and the proportion vaccinated per quarter, the incidence rate ratio (IRR) was lower for children vaccinated with PCV10 (IRR 0.87; CI 95\% 0.76-0.99) and PCV13 (IRR 0.69; CI 95\% 0.60-0.80) compared to no vaccination. A 20\% decreased risk was observed for PCV13 compared to PCV10 (IRR 0.80; Cl 95\% 0.65-0.97). In addition, PCV13 and PCV10 reduced costs with $\$ 594.000$ and $\$ 254.000$ per 100.000 person years.

Conclusion: A decreased risk in admissions due to all-cause pneumonia was observed among children vaccinated by PCVs and this was more strongly pronounced in PCV13. Consequently, cost savings were larger when infants were vaccinated with a 13-valent vaccine.

No conflict of interest

\section{ISPPD-0275}

Pneumococcal Pneumonia---Risky Business

\section{DYSBIOSIS OF UPPER RESPIRATORY TRACT MICROBIOTA IN ELDERLY PNEUMONIA PATIENTS}

W. de Steenhuijsen Piters ${ }^{1}$, E. Huijskens ${ }^{2}$, G. Biesbroek ${ }^{1}$, A. Wyllie ${ }^{1}$, M. van den Bergh ${ }^{3}$, A. van der Ende ${ }^{4}$, R. Veen-

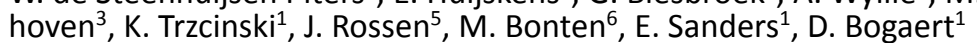

${ }^{1}$ Department of Pediatric Immunology and Infectious Diseases, University Medical Center Utrecht, Utrecht, Netherlands; ${ }^{2}$ Laboratory of Medical Microbiology and Immunology, St. Elisabeth Hospital, Tilburg, Netherlands; ${ }^{3}$ Linnaeus Institute, Spaarne Hospital, Hoofddorp, Netherland; ${ }^{4}$ Department of Medical Microbiology Center for Infection and Immunity Amsterdam, Academic Medical Center, Amsterdam, Netherlands;

${ }^{5}$ Department of Medical Microbiology, University Medical Center Groningen, Groningen, Netherlands; ${ }^{6}$ Department of Medical Microbiology, University Medical Center Utrecht, Utrecht, Netherlands

Background: In elderly, lower respiratory tract infections are a major cause of morbidity and mortality. The most common bacterial pathogen Streptococcus pneumoniae is frequently encountered in the upper respiratory tract as part of the commensal flora. We hypothesized that dysbiosis of the respiratory microbiome is involved in pathogen overgrowth and consequently disease. We therefore compared oropharyngeal microbiota of healthy elderly with elderly pneumonia patients.

Methods: We determined the microbial density and composition of oropharyngeal samples of elderly ( $>60$ years) pneumonia patients $(n=100)$ and healthy elderly $(n=91)$ by barcoded sequencing of the V5-V7 regions of the 16S-rRNA gene.

Results: The overall bacterial density was significantly higher in pneumonia patients compared to controls (median 487.7 vs $15.5 \mathrm{pg} / \mu \mathrm{l}, \mathrm{p}<0.0001$ ). Bacterial diversity was lower in pneumonia patients (Chao1-index 334.0 vs 506.4 species, $\mathrm{p}<0.0001$ ). The most frequently encountered genera were Streptococcus, Rothia, Prevotella, Veillonella, Actinomyces, Neisseria and Lactobacillus. Microbiota profiles differed between healthy elderly and elderly pneumonia patients (Nonmetric Multidimensional Scaling, $p<0.05)$; Streptococcus (pseudo) pneumoniae $(24.9 \%$ vs $9.7 \%$ ) and Rothia dentocariosa (0.10 vs $0.02 \%$ ) showed significantly higher abundances, while lactic acid bacteria like Gemellales (11.2\% vs $27.7 \%$ ) and Prevotella melaninogenica (0.9 vs $6.0 \%$ ) and anaerobes including Veillonela dispar (2.4\% vs $6.1 \%$ ) showed significantly decreased abundances in elderly pneumonia patients compared to healthy controls (FDR-correction, $\mathrm{q}<0.05$ ).

Conclusions: These results suggest that pneumonia in the elderly is accompanied by dysbiosis and bacterial overgrowth of the oropharyngeal microbiome. Whether this imbalance is either a causative factor in the pathogenesis of pneumonia or an epiphenomenon has yet to be determined.

No conflict of interest 


\title{
BURDEN OF DISEASE DUE TO PNEUMONIA AND MENINGITIS IN 1-24 MONTHS AGE GROUP, IN SOUTH INDIA
}

\author{
A. Bose ${ }^{1}$, J. John ${ }^{1}$, V. Balaji ${ }^{2}$, J. Muliyil ${ }^{1}$ \\ ${ }^{1}$ Department of Community Health, Christian Medical College, Vellore, India; ${ }^{2}$ Department of Microbiology, Christian Medical College, Vellore, \\ India
}

In 2005, Christian Medical College recruited a birth cohort, and followed babies up to 2 years of age, to study the incidence of pneumonia and meningitis in this age group. The study collected data on all pneumonia and meningitis including Streptococcus pneumoniae.

Methods: Babies from the cohort were referred to the base hospital with suspected pneumonia or meningitis. Definitions of severe pneumonia and meningitis: were as per WHO guidelines.

Results: 5150 enrolled, with $410(8 \%)$ lost to follow up. 138 cohort babies (cyo = child years of observation) (2.7/100 cyo) cohort were admitted with pneumonia. Pneumonia rates: <6 months of age (6/100 cyo); 6-11 months 2.3 /100 cyo and 1.5/100 cyo in 12-23 months. Radiographically confirmed pneumonia rates: $1.5 / 100$ cyo, $0.3,0.3 / 100$ cyo respectively. $89 \%$ of $x$-rays were WHO readable quality. The lumbar puncture (LP) rate in Vellore: 680/100000 cyo. Total CSF done: 418, of which 312 were infants. 98 had cell count >10/cu mm. 25 more than 100 cells/cu mm. 11 of these had Hib + (47\%); 6 (24\%) were culture positive for S. pneumonia. 22 cohort babies had lumbar punctures. Extrapolating from the cohort: Incidence of "hospitalized pneumonia" $=93 / 2770$ child years /3350 per 100,000 cyo. Incidence of "meningitis" (CSF TC >=10) = 3/2770 child years = 108.5/100,000 cyo.

Carriage study: 302 nasopharyngeal swabs were taken from healthy children, in this age group, 85 (28\%) were positive for Pneumococcus.

Conclusion: These data show that invasive pneumococcal disease is present here in the age group 1-23 months.

No conflict of interest

ISPPD-0217

Pneumococcal Pneumonia---Risky Business

\section{REDUCTION IN COMMUNITY-ACQUIRED ALVEOLAR PNEUMONIA (CAAP) IN CHILDREN AFTER THE INTRODUCTION OF THE PNEUMOCOCCAL CONJUGATED VACCINES (PCVS) IN SOUTHERN ISRAEL}

D. Greenberg ${ }^{1}$, N. Givon-Lavi ${ }^{1}$, S. Ben-Shimol ${ }^{1}$, J. Bar-Ziv², R. Dagan ${ }^{1}$

${ }^{1}$ Pediatric Infectious Disease Unit, Soroka University Medical Center and Ben-Gurion University of the Negev, Beer-Sheva, Israel; ${ }^{2}$ Department of Radiology, Hadassah University Medical Center, Jerusalem, Israel

Background and Aims: PCV7 was introduced to the Israeli vaccination schedule in July 2009 and gradually replaced by PCV13 since November 2010. Our aim was to determine whether hospital visits for radiologically-proven community-acquired alveolar pneumonia (CAAP) among children $<5$ y had been reduced after PCV introduction. Methods: An ongoing prospective population-based study was initiated in 2002, which included all hospital visits (emergency-room or hospitalizations) with WHO radiographically-diagnosed CAAP. Our center is the only medical center in the region, enabling incidence calculation. We defined 3 periods according to vaccination history: 1) prePCV (2004 - 2008; 2) PCV7 (2010 -2011; 70\% of children receiving $\geq 2$ PCV7 doses); 3) PCV13 (2012 - 2013; $\geq 70 \%$ of all children receiving $\geq 2$ PCV13 doses). Vaccine impact was calculated by incidence rate ratio (IRR), adjusted for ethnic group and hospitalization.

Results: 10,142 CAAP cases were enrolled. Annual incidence declined by $14 \%$ and $47 \%$ in the PCV7 and PCV13 periods, respectively (Figure). Significant reductions were observed in all age-groups $<5$ y, especially after PCV13 introduction (Table).

Conclusion: A significant decline in hospital CAAP visits in children $<5$ y was observed after PCV7 introduction with a significant and substantial additional reduction after PCV13 introduction.

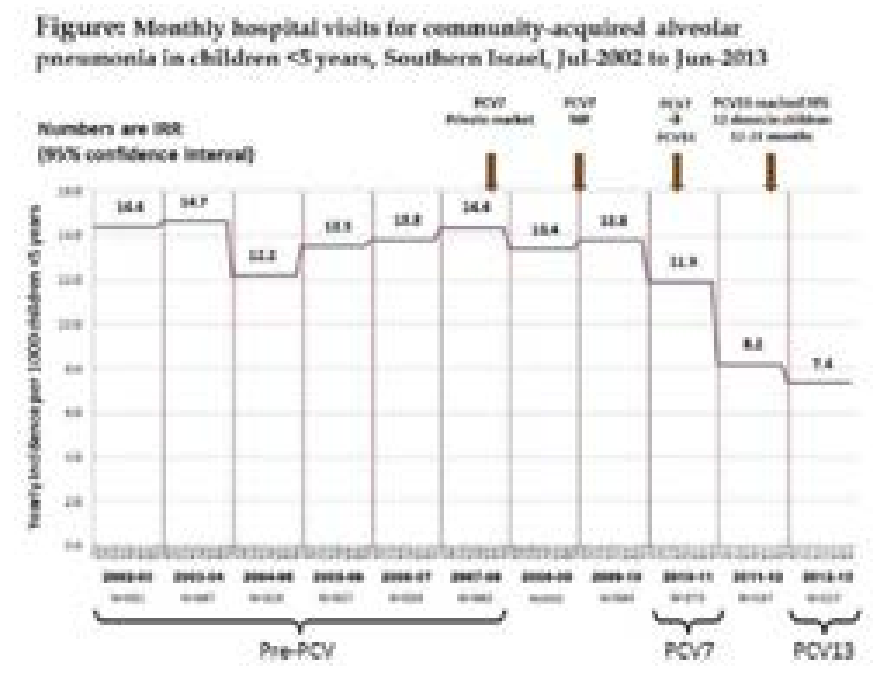


Table: Incidence rate ratio (IRR) of hospital visits for CAAP in childrea $\$ 5 y$, by age group, in Southere Israel: PCV7 and PCV13 periods compared to the pre-PCV period

\begin{tabular}{|c|c|c|c|c|c|c|}
\hline Amest & 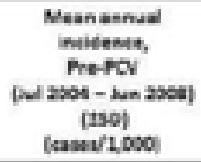 & 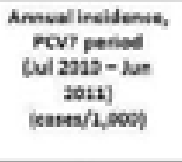 & $\begin{array}{c}\text { novit } \\
\text { nov } \\
\text { maxes }\end{array}$ & 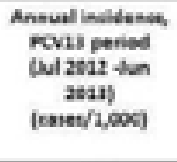 & 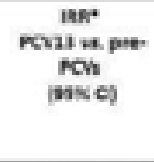 & 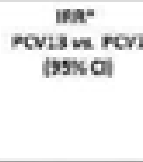 \\
\hline 42 & 2tuts. & 245 & $0.56(0.83 .211)$ & 139 & $0.56(0.47-0.46)$ & 0.58 [0.45-0.69] \\
\hline 12.23 & 20.1n:.5 & 24: & Q.70 (0.59-0.84) & 9.7 & $0.48(0.49-0.58)$ & Q.6s [0.56-0.54] \\
\hline 2453 & 8.250 .7 & 73 & $4.89(0.72-04)$ & 4,4 & $0.44(0.45-9.4)$ & 0.60 os:- 0.7$]$ \\
\hline Oreral os $y$ & 13.sel. & 119 & e. & 2.4 & $0.33(0.45-0.93)$ & 0.61 (0.95-0.69) \\
\hline
\end{tabular}

Conflict of interest

ISPPD-0225

Pneumococcal Pneumonia---Risky Business

\section{DISTRIBUTION OF PNEUMOCOCCAL SEROTYPES DETECTED THROUGH URINE ANALYSIS AMONG US ADULTS HOSPITALIZED WITH PNEUMONIA AFTER INTRODUCTION OF PCV13}

C.G. Grijalva ${ }^{1}$, R.G. Wunderink ${ }^{2}$, D. Williams ${ }^{3}$, Y. Zhu ${ }^{4}$, R. Balk ${ }^{5}$, S. Fakhran ${ }^{6}$, M. Courtney ${ }^{2}$, E. Anderson ${ }^{7}$, C. Qi ${ }^{8}$, L. Hicks ${ }^{9}$, C.G. Whitney ${ }^{9}$, M.R. Moore ${ }^{9}$, S. Jain ${ }^{9}$, K.M. Edwards ${ }^{3}$, W.H. Self ${ }^{10}$

${ }^{1}$ Health Policy, Vanderbilt University, Nashville, USA; ${ }^{2}$ Medicine, NorthWestern University, Chicago, USA; ${ }^{3}$ Pediatrics, Vanderbilt University, Nashville, USA; ${ }^{4}$ Biostatistics, Vanderbilt University, Nashville, USA; ${ }^{5}$ Medicine, Rush University, Chicago, USA; ${ }^{6}$ Medicine, John H. Stroger Jr. Hospital, Chicago, USA; ${ }^{7}$ Pediatrics and Medicine, Emory University, Atlanta, USA; ${ }^{8}$ Pathology, NorthWestern University, Chicago, USA; ${ }^{9}$ National Center for Immunization and Respiratory Diseases, CDC, Atlanta, USA; ${ }^{10}$ Emergency Medicine, Vanderbilt University, Nashville, USA

Background: Whether PCV13 should be recommended for adults is unclear. We assessed the distribution of pneumococcal serotypes using Pfizer's serotype-specific urinary antigen detection assay (UAD) among adults enrolled in the Center for Immunization and Respiratory Diseases (CDC) Etiology of Pneumonia in the Community (EPIC) study, after initiation of infant vaccination with PCV13 in 2010.

Methods: Adults ( $>18$ years) hospitalized with community-acquired pneumonia and enrolled from January 2010June 2012 were included. Infections due to PCV13 serotypes were detected using UAD. We compared serotype distributions between consecutive respiratory seasons (July-June) for all adults, adults living with young children, and those with chronic comorbidities.

Results: Among 2044 adults with UAD results (82\% of all enrolled), median age was 58 years (IQR: 47-71). Overall, PCV13 serotypes were detected in 178 (9\%) adults, including 40 (2\%) with PCV7 serotypes. The most common serotypes were 19A (31\%), $5(20 \%), 3(17 \%)$ and $7 F(15 \%)$. Twenty adults (11\%) had $>1$ serotype detected, with fifteen including serotype 5 . The number of pneumonia cases dropped between consecutive seasons. Declines in the proportion caused by PCV13 serotypes were significant only among adults living with children (Table).

Conclusion: Most pneumococcal detections using UAD were due to serotypes covered only by PCV13 and not PCV7. Changes between consecutive seasons suggest reductions in pneumonia may be occurring among adults due to PCV13 herd effects.

\begin{tabular}{|c|l|l|}
\hline & July 2010 - June 2011 & July 2011 - June 2012 \\
\hline All adults with pneumonia & $\mathrm{N}=1031$ & $\mathrm{~N}=783$ \\
\hline PCV13-serotypes & $93(9 \%)$ & $62(8 \%)$ \\
\hline Serotypes only in PCV13 & $77(7 \%)$ & $52(7 \%)$ \\
\hline Living with young children & $\mathrm{N}=109$ & $\mathrm{~N}=65$ \\
\hline PCV13-serotypes & $16(15 \%)$ & $6(9 \%)$ \\
\hline Serotypes only in PCV13 & $14(13 \%)$ & $4(6 \%)^{*}$ \\
\hline With chronic comorbidities & $\mathrm{N}=840$ & $\mathrm{~N}=631$ \\
\hline PCV13-serotypes & $72(9 \%)$ & $48(8 \%)$ \\
\hline Serotypes only in PCV13 & $58(7 \%)$ & $43(7 \%)$ \\
\hline
\end{tabular}

$* p=0.04$, chi-square test

Conflict of interest 
ISPPD-0483

Pneumococcal Pneumonia---Risky Business

\title{
CHILD MORTALITY AFTER DISCHARGE FOLLOWING SUSPECTED PNEUMONIA, MENINGITIS OR SEPSIS IN RURAL GAMBIA
}

\author{
A.V. Chhibber ${ }^{1}$, P. Hill' ${ }^{1}$, J. Jafali ${ }^{2}$, M. Jasseh ${ }^{3}$, M.I. Hossain ${ }^{4}$, M. Ndiaye ${ }^{4}$, J.C. Pathirana ${ }^{4}$, B. Greenwood ${ }^{5}$, G. Mackenzie ${ }^{4}$ \\ ${ }^{1}$ Centre for International Health, School of Medicine University of Otago Dunedin NZ, Dunedin, New Zealand; ${ }^{2}$ Statistics, Medical Re- \\ search Council The Gambia Unit, Fajara, Gambia; ${ }^{3}$ Disease Control and Elimination Theme, Medical Research Council The Gambia Unit, Fajara \\ Gambia; ${ }^{4}$ Child Survival Theme, Medical Research Council The Gambia Unit, Fajara, Gambia; ${ }^{5}$ Faculty of Infectious and Tropical Diseases, Lon- \\ don School of Hygiene \& Tropical Medicine, London, United Kingdom
}

Background and Aims: We determined the risk of mortality following discharge from hospital of Gambian children with suspected pneumonia, sepsis, or meningitis.

Methods: Children were resident in the Basse Health and Demographic Surveillance System (BHDSS), aged 2-59 months, diagnosed with suspected pneumonia, sepsis, or meningitis, admitted at Basse Health Centre between May 2008 and April 2012, and discharged alive. Children were followed for 180 days after discharge. Vital status was monitored by the BHDSS. Multivariable models described mortality associated with a) clinical syndrome on admission and $b$ ) pre-discharge risk factors.

Results: 3735 children were eligible for analysis. 105 died during follow-up; half within the first 45 days. Mortality was associated positively or negatively with age (Hazard ratio [HR] 0.98 [95\% $\mathrm{Cl}: 0.96,0.99]$ for every month increase), pneumonia with severe malnutrition ( $\mathrm{HR} 8.74$ [95\% Cl: 4.93, 15.49]), meningitis with severe malnutrition (HR 13.90 [95\% Cl: 5.43, 35.58]) and sepsis with severe malnutrition (HR 18.79 [95\% Cl: 11.65, 30.32]). Risk factors for mortality were: neck stiffness (HR 17.60 [95\% Cl: 7.36, 42.10]); mid-upper arm circumference $<10.5 \mathrm{~cm}(\mathrm{HR}$ 11.52 [4.59, 28.90]); severe malnutrition (HR 3.94 [95\% Cl: $2.11,7.36]$ ); non-medical discharge (HR 6.22 [95\% Cl: 2.98, 13.01]); dry season (HR 2.33 [95\% Cl: 1.44, 3.77]); oxygen saturation (HR 0.95 [95\% Cl: 0.93, 0.98] per percent increase); haemoglobin concentration (HR $0.82[95 \% \mathrm{Cl}: 0.74,0.90]$ ) per $\mathrm{g} / \mathrm{dL}$ increase); and temperature (HR 0.70 $[0.58,0.84]$ per ${ }^{\circ} \mathrm{C}$ increase).

Conclusion: Post-discharge mortality is significant in rural Gambia and associated particularly with malnutrition. Children at high risk of post-discharge mortality should be followed carefully.

No conflict of interest

ISPPD-0348

Pneumococcal Pneumonia---Risky Business

\section{SEROTYPE, SYNDROME DISTRIBUTION AND ANTIMICROBIAL SUSCEPTIBILITY OF ISOLATES FROM INVASIVE PNEUMOCOCCAL DISEASE (IPD) IN CHILDREN <5 YEARS IN INDIA}

A. Manoharan ${ }^{1}$, Alliance for Surveillance of Invasive Pneumococci $\{$ ASIP $\}$ Study Group ${ }^{1}$, M. Deloria- Knoll ${ }^{2}$, G.D. Kahn², K.L. O' Brien²

${ }^{1}$ Medicine Unit I and Infectious Diseases, Christian Medical College, Vellore, India; ${ }^{2}$ International Vaccine Access Center, The Johns Hopkins Bloomberg School of Public Health, Baltimore, USA

Background and Aims: Information on serotypes causing IPD among children in India is limited. We aimed to determine serotype prevalence, syndrome distribution and antimicrobial susceptibility pattern of IPD cases among children $<5$ years across multiple geographic sites in India.

Methods: The Alliance for Surveillance of Invasive Pneumoccoci (ASIP) network (January 2011-August 2013) included 10 states with 18 tertiary care centers/teaching institutes laboratories, 59 sentinel pediatricians, and 10 central laboratories participating. The network identified IPD cases through prospective enrollment of clinically suspected IPD cases and through prospective and retrospective laboratory surveillance for IPD isolates. Children $<5$ years with clinical symptoms suggestive of pneumonia, meningitis or bacteremia had blood, cerebrospinal fluid or other normally sterile body fluids cultured based on clinical judgment and ASIP recommendations. Pneumococcal isolates were serotyped by Quellung method and antimicrobial susceptibility determined.

Results: Of 237 IPD cases identified from both prospective and lab surveillance predominant serotypes were 14 (15\%), $5(14 \%), 1$ (11\%), 19F (10\%), 6B (6\%) and 19A (5\%). Penicillin and cefotaxime non-susceptibility was $8 \%$ and $12 \%$. Cotrimoxazole and erythromycin resistance was $37 \%$ and $58 \%$. Of 164 IPD cases with clinical characterization, $41.5 \%$ had meningitis, $53.7 \%$ had pneumonia and $4.9 \%$ had another syndrome. Of 3146 clinically suspected cases evaluated prospectively, $2.0 \%$ had pneumococci isolated; mortality was $2.6 \%$ overall and $9.4 \%$ among IPD cases. Conclusion: IPD surveillance across India found $\sim 70 \%$ of IPD isolates are included in the currently licensed, available PCVs. This is key baseline data to monitor post-introduction impact if PCVs are introduced in the Indian EPI.

Conflict of interest 
ISPPD-0231

Pneumococcal Pneumonia---Risky Business

\title{
BETALACTAM TREATMENT UNTIL NO FEVER FOR 48HOURS (AT LEAST 5 DAYS) VERSUS 10 DAYS IN COMMUNITY- ACQUIRED PNEUMONIA: RANDOMISED, NON-INFERIORITY, OPEN STUDY
}

\author{
K. Strålin' ${ }^{1}$ A. Rubenson ${ }^{2}$, H. Lindroth ${ }^{3}$, S. Hagberg ${ }^{4}$, L. Bodin 5 , G. Stendlund ${ }^{3}$, E. Sandholm², H. Holmberg ${ }^{6}$ \\ ${ }^{1}$ Department of Infectious Diseases, Karolinska University Hospital, Stockholm, Sweden; ${ }^{2}$ Department of Infectious Diseases, Karlstad Hospital, \\ Karlstad, Sweden; ${ }^{3}$ Department of Infectious Diseases, Eskilstuna Hospital, Eskilstuna, Sweden; ${ }^{4}$ Department of Infectious Diseases, Falun Hos- \\ pital, Falun, Sweden; ${ }^{5}$ Institute of Environmental Medicine, Karolinska Institute, Stockholm, Sweden; ${ }^{6}$ Department of Infectious Diseases, Öre- \\ bro University Hospital, Örebro, Sweden
}

Background and Aims: The optimal duration of antibiotic treatment for community-acquired pneumonia (CAP) is sparsely studied. In an open randomized controlled study, we aimed to compare beta lactam treatment until no fever for $48 \mathrm{~h}$ (at least $5 \mathrm{~d}$; short course therapy) with standard therapy (10 d).

Methods: In a prospective multicentre study at Swedish infectious diseases departments, patients with radiologically confirmed, uncomplicated febrile CAP were randomised to receive short course therapy or standard therapy (study register no. ISRCTN14523624). For statistical equivalence, we accepted a cure rate difference of 5\% including $95 \%$ confidence intervals $(\mathrm{Cl})$.

Results: Of 207 enrolled patients, adverse events were noted in 4 patients with standard therapy (diarrhoea $n=3$; oral candida infection $n=1$ ). The per protocol analysis included 88 patients with short course therapy and 86 patients with standard therapy. Clinical cure was noted in $90 \%$ with short course therapy and $94 \%$ with standard therapy, which yields an $11 \%$ advantage for standard therapy including $95 \% \mathrm{Cl}$. However, among 64 patients with pneumococcal aetiology, high similar rates of clinical cure were noted in those with short course therapy (96\%; $27 / 28)$ and standard therapy $(97 \% ; 35 / 36)$.

Conclusion: In the present study, statistical equivalence between short course therapy and standard therapy for CAP cure could not be found. However, in patients with pneumococcal aetiology, high cure rate with short course therapy was noted. This supports use of short course beta lactam therapy in patients with pneumococcal pneumonia and encourages efforts to detect pneumococcal aetiology in CAP.

No conflict of interest

ISPPD-0401

Pneumococcal Pneumonia---Risky Business

\section{INCIDENCE AND SEASONALITY OF PNEUMOCOCCAL PNEUMONIA IN TONE DISTRICT, TOGO 2010-2013}

H. Tall ${ }^{1}$, M.S. Makawa ${ }^{2}$, K. Agbenoko ${ }^{3}$, T.S. Tamekloe ${ }^{4}$, M. Amidou ${ }^{5}$, E. Espié6 ${ }^{\text {, B.M. Njanpop-Lafourcade }}$, J.E. Mueller ${ }^{8}$, B.D. Gessner ${ }^{9}$, J.C. Moïsi ${ }^{9}$

${ }^{1}$ Epidemiology, Agence de Médecine Préventive, Ouagadougou, Burkina Faso; ${ }^{2}$ Direction Provinciale de la Santé de Tône, Ministry of Health Togo, Dapaong, Togo; ${ }^{3}$ Regional Hospital Laboratory, Ministry of Health Togo, Dapaong, Togo; ${ }^{4}$ Epidemiology Division, Ministry of Health Togo, Lomé, Togo; ${ }^{5}$ Direction Regionale de la Santé des Savanes, Ministry of Health Togo, Dapaong, Togo; ${ }^{6}$ Epidemiology, Institut Pasteur, Dakar, Senegal ${ }^{7}$ Laboratory Expertise, Agence de Médecine Préventive, Paris, France; ${ }^{8}$ Epidemiology, Ecole des Hautes Etudes en Santé Publique, Paris, France ${ }^{9}$ Epidemiology, Agence de Médecine Préventive, Paris, France

Background and Aims: We aimed to document the burden and characteristics of pneumococcal pneumonia in the African meningitis belt.

Methods: From May 2010 to April 2013, we enrolled patients admitted for clinical pneumonia at the five hospitals in Tone District, northern Togo. We obtained blood for culture and real-time lytA PCR. Chest X-rays (CXR) read according to standardized WHO methodology identified patients with lobar consolidation or pleural effusion.

Results: Among 1550 patients (median age 26 years), 26\% had severe pneumonia and 39\% a positive CXR. Clinical, severe and CXR-confirmed pneumonia rates were 167, 45 and 63 per 100,000 and highest among persons $<1$ and $30+$ years of age. Annual peaks in incidence occurred during the dry season in persons age $5+$. In children $<5$, severe and CXR-confirmed pneumonia rates decreased over the study period and disease seasonality was less evident. Bacteremic pneumococcal pneumonia accounted for $4.7 \%$ of cases $(n=72)$, with culture yields increasing with age. In addition, $7 \%$ of culture-negative cases were lytA-positive (65/895, testing ongoing). PCV13 serotypes accounted for $90 \%$ of isolates (35/39) and 79\% of PCR-positive cases (48/55, serotyping ongoing). Serotypes 3, 1 and 5 were the most common representing $13.9 \%(n=19), 10.2 \%(n=14)$ and $8.8 \%(n=12)$ of cases, respectively.

Conclusion: This study is the first to document the burden and characteristics of pneumococcal pneumonia in the African meningitis belt showing high incidence across all age groups, a seasonality similar to meningitis among adults, and excellent coverage for PCV13. A 3-year PCV13 impact study is planned.

Conflict of interest 
ISPPD-0040

Pneumococcal Pneumonia---Risky Business

\section{THE PREDICTIVE VALUE OF IL6 TO PNEUMOCOCCAL COMMUNITY-ACQUIRED PNEUMONIA}

A.G. Vasconcellos ${ }^{1}$, J. Clarêncio ${ }^{2}$, D. Andrade ${ }^{2}$, M.R.A. Cardoso ${ }^{3}$, A. Barral' ${ }^{2}$, C.M. Nascimento-Carvalho ${ }^{1}$

${ }^{1}$ Health Sciences PhD Program (PPgCS), Federal University of Bahia School of Medicine, Salvador, Brazil; ${ }^{2}$ Centro de Pesquisa Gonçalo Muniz, Fundação Oswaldo Cruz, Salvador, Brazil; ${ }^{3}$ Epidemiology, São Paulo University School of Public Health, Sao Paulo, Brazil

Background and Aims: Community-acquired pneumonia (CAP) is the main cause of death in children under 5 years worldwide and Streptococcus pneumoniae is the commonest bacterial agent. However, it is difficult to identify pneumococcal CAP. We aimed to evaluate if there is association between any cytokine, on admission, and pneumococcal CAP.

Methods: This was a prospective study conducted at the Emergency Room of Federal University of Bahia Hospital, in Salvador, Brazil. Children aged $<5$ years admitted with CAP from September/2003 to May/2005 were evaluated. On admission, clinical and radiological data were collected as well as biological samples to investigate 18 etiological agents and determine serum cytokine dosage (IL8, IL6, IL10, IL1 $\beta$, IL12, TNF $\alpha$, IL4, IL5, IFNץ, IP10, RANTES, and MCP1).

Results: From 219 enrolled patients, 127 (58\%) were boys and the mean age was $20 \pm 15$ months. Pneumococcal infection was detected in $48(23 \%)$ cases among which the median IL6 concentration was $26 \mathrm{pg} / \mathrm{ml}$ (interquartile range:2-60pg/ml). Other causative agents (Haemophilus influenzae, Moraxella catarrhalis, atypical bacteria, viruses, mixed) were detected in 171 cases when the median IL6 concentration was $0 \mathrm{pg} / \mathrm{ml}$ (interquartile range: 0-11 pg/ $\mathrm{ml}$ ). The area under the ROC curve for IL6 to predict pnemococcal CAP was $0.72(p=0.04)$. By multivariate analyses, with pneumococcal CAP as dependent variable, IL6 was independent predictor for pneumococcal infection (OR=4; $p$ $<0.001 ;$ IC95\%=2-7; cutoff point $11 \mathrm{pg} / \mathrm{ml}$ ).

Conclusion: IL6 is an independent predictor for pneumococcal CAP.

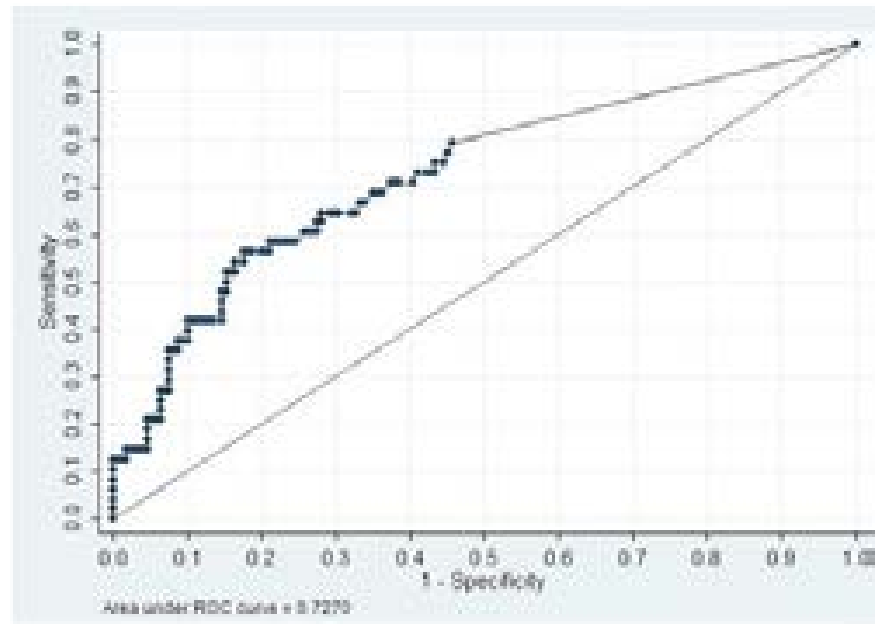

No conflict of interest

Poster Abstracts

ISPPD-0003

Pneumococcal Pneumonia---Risky Business

\section{INCIDENCE AND TREND OF ANTIBIOTIC RESISTANCE AMONG STREPTOCOCCUS PNEUMONIAE ISOLATED FROM PEDIATRICS IN EKITI AND ONDO, STATES OF SOUTHWESTERN NIGERIA}

V.A. Ajibade ${ }^{1}$

${ }^{1}$ Science Technology (Microbiology), The Federal Polytechnic, Ado-Ekiti, Nigeria

The incidence of Streptococcus pneumoniae isolated from pediatrics admitted into various hospitals in Ekiti and Ondo States from the months of January 2009 to July 2010 were investigated. It was observed that out of the 400 samples collected from children in both states, $57(14 \%)$ were positive for the isolates, while $295(74 \%)$ were negative. Indicating a low incidence in both states but higher in Ondo State. Out of the 57 positive samples, 44 $(77 \%)$ were isolates from sputum, $4(7 \%)$ from urine, $1(2 \%)$ from blood and $8(14 \%)$ from ear swab. The highest number of positive isolates were $41(68 \%)$ from males while $16(32 \%)$ were from females. From sputum, 33 $(97 \%)$ were susceptible to ciprofloxacin and $5(14 \%)$ to loxacillin. From urine, $3(75 \%)$ isolates were susceptible to 
ofloxacin. The isolates from blood samples were susceptible to both ciprofloxacin and gentamicin. The isolates from ear swab were susceptible to erythromycin and ciprofloxacin but 1 (50\%) was susceptible to ofloxacin, clindamycin, gentamicin, cotrimoxazole ampicilin and cloxacillin. All isolates from urine samples showed resistance to majority of the antibiotics. All isolates from blood showed resistance to all antibiotics tested except ciprofloxacin and gentamicin. Two isolates from ear swab showed resistance to ceftriaxone and augmentin. The most active antibiotics tested were ciprofloxacin (92\%), gentamycin (78\%), erythromycin (70\%), ofloxacin (60\%) and clindamycin (46\%).

Conflict of interest

ISPPD-0080

Pneumococcal Pneumonia---Risky Business

\title{
THE IMPACT OF PCV7/PCV13 CHILDREN'S VACCINATION ON THE INCIDENCE OF PNEUMONIA MORBIDITY IN KIELCE, POLAND IN A 7-YEAR OBSERVATION PERIOD
}

\author{
M. Patrzalek ${ }^{1}$, P. Gorynski ${ }^{2}$, M. Kotowska ${ }^{3}$, P. Albrecht ${ }^{3}$
}

${ }^{1}$ NZOZ, "Promed", Kielce, Poland; ${ }^{2}$ National Institute of Hygiene, National Institute of Public Health, Warsaw, Poland; ${ }^{3}$ Department of Paediatric Gastroenterology and Nutrition, Medical University of Warsaw, Warsaw, Poland

Background and Aims: In 2006, the city of Kielce, Poland introduced PCV7 (since 2011, PCV13) mandatory vaccination programme for children $<2$ years of age. The aim of this study was to analyse the direct and indirect effects of PCV7/ PCV13 vaccination on pneumonia incidence rate in the 7-year follow-up period in comparison to the city of Ostrowiec Swietokrzyski, where there was no mandatory vaccination scheme present.

Methods: PCV7/PCV13 vaccines were given in a $2+1$ scheme. The compliance rate for vaccinations reached approx. $99 \%$. The following age groups were analysed: 0-1, 30-49, 50-65 and 65+ years in a period of 2006-2012. CochranArmitage test investigated the significance of the trend in pneumonia morbidity. The significance of deviations was tested. The importance of the trend (in case of deviations from linearity) was confirmed by Mantel test.

Results: The greatest decrease in pneumonia morbidity in analysed period was observed for children $<2$ years of age: $96.5 \%(2005,25 / 1000 ; 2012,0.88 / 1000)$. In the $65+$ age group, there was a $66.5 \%$ decline for all diagnosed pneumonia, followed by lower, also statistically significant, decline in other age groups: $30.8 \%(30-49 \mathrm{y})$ and $56.8 \%$ (50-64 y). The decreasing trend continued for seven consecutive years of observation. We demonstrated also a statistically significantly higher rate of pneumonia incidence among all age groups in the city, Ostrowiec Swietokrzyski, not conducting mass pneumococcal vaccination program.

Conclusion: The results clearly proved, with a statistical significance, direct and indirect effectiveness on pneumonia prevention due to PCV7/PCV13 mass vaccination programme.

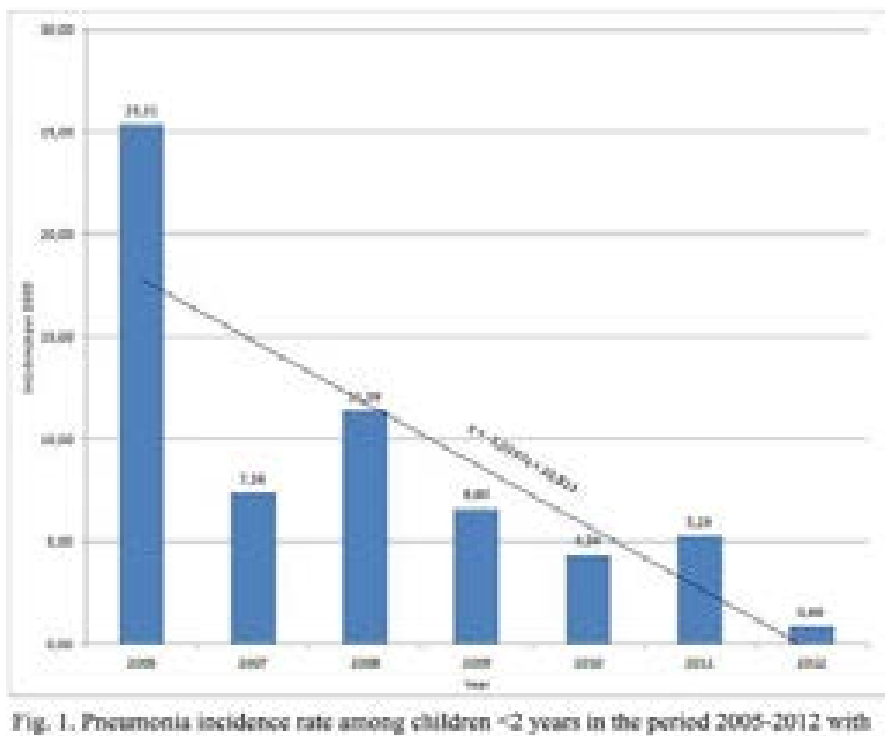

rig. 1. Fnetancnia iscldence rate among children - 2 years in the porisd $2005-2012$ witt regession lite. 


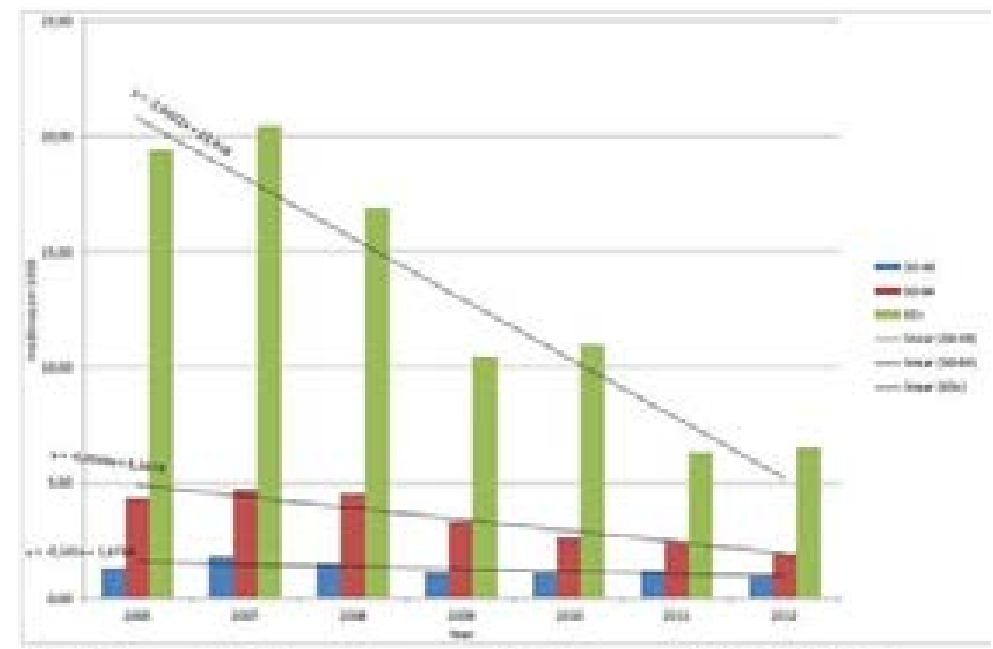

Fig.2. Pheumceis incidence rate among analyred age geoups $(30-49,50-64,65+)$ in the years 2005,2012 with regression lines.

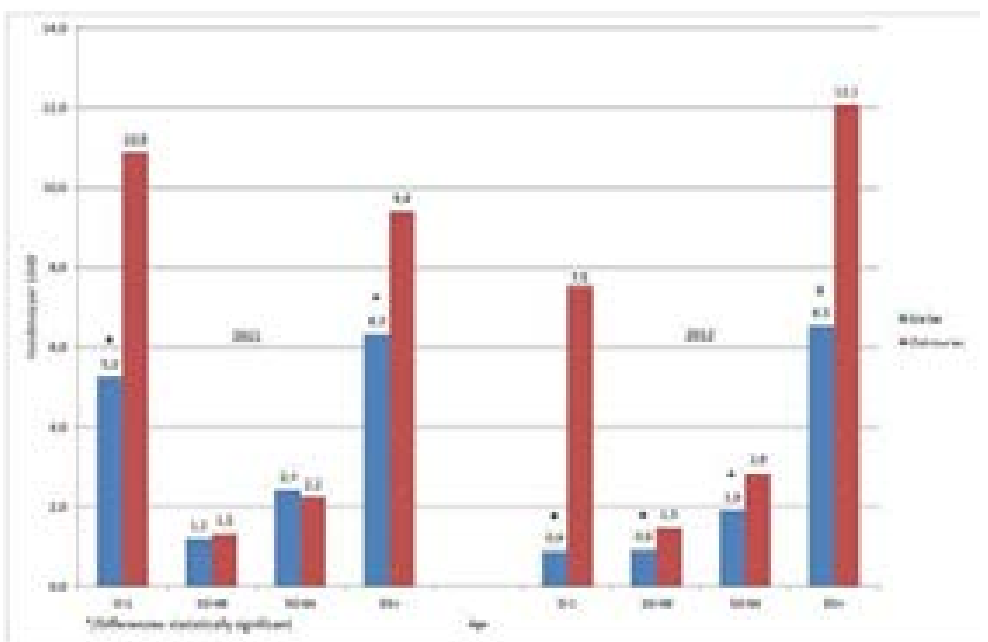

Fis. 3. Compariwen of preumonia incidence rate amons analyrad age groups $(0.1,30-49$ 50-64, 65t) in Kislec and Ostrowies Swictokreyski in the years 2011 and 2012.

Conflict of interest

ISPPD-0287

Pneumococcal Pneumonia---Risky Business

\section{ACTIVE SURVEILLANCE OF PNEUMOCOCCAL DISEASE IN ELDERLY: POTENTIAL EFFECT OF PCV13 INTRODUCTION ON EMERGENCY DEPARTMENT ACCESSES FOR LOWER RESPIRATORY TRACT INFECTIONS}

F. Ansaldi ${ }^{1}$, A. Orsi ${ }^{2}$, D. De Florentiis ${ }^{3}$, S. Schiaffino ${ }^{4}$, V. Turello ${ }^{5}$, R. Rosselli ${ }^{5}$, R. Carloni ${ }^{6}$, P. Canepa ${ }^{7}$, A. Ceravolo $^{7}$, L. Sticchi ${ }^{7}$, R. Zanetti ${ }^{7}$, I. Cremonesi ${ }^{7}$, P. Brasesco ${ }^{7}$, P. Moscatelli ${ }^{7}$, P. Durando ${ }^{1}$, G. Icardi $^{1}$

${ }^{1}$ Department of Health Sciences (DiSSal), IRCCS-AOU San Martino-Istituto Nazionale per la Ricerca sul Cancro, Genoa, Italy; ${ }^{2}$ Department of Health Sciences (DiSSal), University of Genoa, Genoa, Italy; ${ }^{3}$ Office of Maritime Health Air and Border (USMAF), Ministry of Health, Genoa, Italy; ${ }^{4}$ Department of Health and Social Services - Prevention Public Health and Vulnerable Social Groups, Liguria Region, Genoa, Italy ${ }^{5}$ Local Health Unit, ASL ${ }^{3}$ Genovese, Genoa, Italy; ${ }^{6}$ Health Regional Agency ARS Liguria, Liguria Region, Genoa, Italy; ${ }^{7}$ Ligurian Pneumococcal Study Group, Liguria Region, Genoa, Italy

Background and Aims: In Liguria, an administrative region in Northern Italy characterized by a decade of high PCV coverage in paediatric age group, new PCV13 recommendations for free active immunisation in adults with risk factors and subjects aged $\geq 70$ years old have been issued. Main aims of this study are (i) a descriptive epidemiology of the clinical burden of lower respiratory tract infections (LRTI) in adults $\geq 18$ years of age, and (ii) a cross-over evaluation of the effect of introduction and increasing implementation of PCV13 vaccination in adults aged $\geq 70$ years old, in terms of emergency department (ED) accesses for LRTI, obtained by a Syndrome Surveillance System (SSS).

Methods: A population cohort of $>430,000$ individuals aged $\geq 18$ years old will be actively surveyed for 60 months, by an ED access, chief complaint based SSS, designed for the detection and analysis of ED accesses for LRTI. Results: During pre-PCV period, annual cumulative incidence of ED accesses for LRTI was equal to 7/1000 and 
$2 \%$ in $\geq 65$ and $\geq 85$ year adults, respectively. In $\geq 65$ years adults, more than $70 \%$ of subjects identified by the SSS has at least one risk condition, with a peak of $87 \%$ in $\geq 85$ year cohort. New Ligurian PCV13 recommendations can potentially reach more than $75 \%$ of ED accesses for LRTI.

Conclusion: Data highlights the heavy impact of LRTI in terms of ED accesses, especially in the elderly and subjects with chronic conditions and the usefulness of SSS tool for monitoring PCV vaccination effect.

Conflict of interest

ISPPD-0025

Pneumococcal Pneumonia---Risky Business

\title{
CARDIAC MICROLESIONS FORM IN A CbPA-DEPENDENT MANNER DURING INVASIVE PNEUMOCOCCAL DISEASE
}

A.O. Brown ${ }^{1}$, B. Mann ${ }^{2}$, G. Gao ${ }^{2}$, P. Faverio ${ }^{3}$, M.I. Restrepo ${ }^{4}$, G.V. Halade ${ }^{5}$, E.M. Mortensen ${ }^{6}$, M.L. Lindsey ${ }^{7}$, M. Hanes ${ }^{8}$, K.I. Happel ${ }^{9}$, S. Nelson ${ }^{9}$, G.J. Bagby ${ }^{9}$, E.I. Tuomanen ${ }^{2}$, C.J. Orihuela ${ }^{1}$

${ }^{1}$ Microbiology \& Immunology, South Texas Research Facility-The University of Texas Health Science Center at San Antonio, San Antonio, USA;

${ }^{2}$ Infectious Diseases, St. Jude Children's Research Hospital, Memphis, USA; ${ }^{3}$ Respiratory Medicine, University of Milan Bicocca, Monza, Italy

${ }^{4}$ Medicine, Veterans Health Care System and University of Texas Health Science Center at San Antonio, San Antonio, USA; ${ }^{5}$ Medicine, The University of Alabama at Birmingham, Birmingham, USA; ${ }^{6}$ Internal Medicine, University of Texas Southwestern Medical Center, Dallas, USA;

${ }^{7}$ Physiology and Biophysics, University of Mississippi Medical Center, Jackson, USA; ${ }^{8}$ Laboratory Animal Resources, The University of Texas Health Science Center at San Antonio, San Antonio, USA; ${ }^{9}$ Physiology and Section of Pulmonary/Critical Care Medicine, Louisiana State University Health Sciences Center, New Orleans, USA

Individuals hospitalized for invasive pneumococcal disease (IPD) frequently experience adverse cardiac events. Herein we describe previously unrecognized cardiac microlesions formed within the myocardium during experimental Streptococcus pneumoniae infection in mice and rhesus macaques. In mice, microlesion formation was positively correlated with bacterial burden in the blood and serum levels of troponin, a marker for cardiac damage. Microlesions were accompanied by progressive changes in cardiac electrophysiology, as measured by limb-lead ECG, which indicated the development of aberrant contractility. Microlesions increased in number and size during IPD, were confined to the heart, and lacked infiltrating immune cells. Bacterial translocation across the cardiac vasculature into heart tissue required the pneumococcal adhesin CbpA and the host-ligands laminin receptor and platelet activating factor receptor. Once in the myocardium, pneumococci remained extracellular. The pore forming toxin pneumolysin was present at sites of cell death and was determined to be required for microlesion formation. Immunization with a fusion construct of $\mathrm{CbpA}$ peptides and a pneumolysin toxoid protected against microlesion formation. We conclude that cardiac microlesions form during IPD and contribute towards aberrant cardiac electrophysiology. Because microlesions were also observed in non-human primates, they help to explain the high incidence of adverse cardiac events seen in humans with IPD.

No conflict of interest

ISPPD-0519

Pneumococcal Pneumonia---Risky Business

\section{RISK FACTORS FOR CHILD PNEUMONIA IN SOUTH-EAST ASIA - A SYSTEMATIC REVIEW AND META-ANALYSIS}

\author{
D. Bassani ${ }^{1}$, K. Wazny $^{1}$, C. McPhail ${ }^{1}$, T. Sivananthajothy ${ }^{1}$, M. Santosham ${ }^{2}$
}

${ }^{1}$ Centre for Global Child Health, The Hospital for Sick Children, Toronto, Canada; ${ }^{2}$ IVAC, John's Hopkins Bloomberg School of Public Health, Toronto, Canada

Introduction: Pneumonia is the leading killer of children under five worldwide, causing 1.1 million deaths in 2012. Pneumonia deaths are highly concentrated in Asia and Africa. Deaths from pneumonia have decreased by $35 \%$ since 2000, but further reductions are likely possible. As part of the Diarrhoea and Pneumonia Evidence Synthesis Project, we systematically reviewed risk factors for diarrhoea and pneumonia in South East Asia (India, Pakistan, Nepal, Sri Lanka and Bangladesh).

Methods: EMBASE, Medline, AMED and the WHO Eastern Mediterranean and Southeast Asian databases were searched and titles and abstracts of 4,576 articles were reviewed in duplicate. We included 140 papers in the analysis for risk factors the incidence, severity or duration of diarrhoea or pneumonia.

Results and Conclusion: There were no significant differences in pneumonia incidence by child's age or gender. Children who were malnourished had an increased risk for pneumonia and pneumonia mortality (RR: $6.59,95 \% \mathrm{Cl}$ : 2.49 to 17.43 and RR: $5.08,95 \% \mathrm{Cl}: 3.44$ to 7.50 , respectively). Incompletely immunization is associated with an increased pneumonia incidence (RR: $2.50,95 \% \mathrm{Cl}: 1.46$ to 4.28). While children who live in crowded households have higher pneumonia incidence (RR: 1.11, 1.01 to 1.75), there were no differences in pneumonia mortality or severity. Use of non-clean fuel sources significantly increased pneumonia incidence (RR: $1.36,95 \% \mathrm{Cl}: 1.05$ to 1.57 ). Breastfeeding was significantly protective against pneumonia, reducing its incidence. Higher maternal and paternal education were associated with lower pneumonia incidence (RR: $0.71,95 \% \mathrm{Cl}: 0.58$ to 0.87 and RR: $0.64,0.53$ to 0.77 , respectively). Socioeconomic status was not a significant risk factor for pneumonia incidence. Importantly, the varied criteria used to define Pneumonia in each study significantly influenced the associations estimated in this meta-analysis.

No conflict of interest 
ISPPD-0206

Pneumococcal Pneumonia---Risky Business

\title{
SEROTYPE PREVALENCE OF STREPTOCOCCUS PNEUMONIAE IN SURVEILLANCE OF INVASIVE DISEASES IN CHILDREN UNDER 5 YEARS, OF EASTERN INDIA
}

\author{
A. Ghosh ${ }^{1}$, N. Bhattacharyya ${ }^{1}$, M. Mitra ${ }^{1}$, M. Nandy ${ }^{2}$, J. Choudhury ${ }^{1}$, B. Acharya ${ }^{3}$, J. Basu ${ }^{4}$, A. Agarwal ${ }^{5}$ \\ ${ }^{1}$ Pediatric Medicine, Institute of Child Health, Kolkata, India; ${ }^{2}$ Pediatric Medicine, Midnapore Medical College, Midnapore, India \\ ${ }^{3}$ School of Medical Science and Technology, IITKharagpur, Kharagpur, India; ${ }^{4}$ Microbiology, Institute of Child Health, Kolkata, India \\ ${ }^{5}$ Clinical Microbiology, Belle Vue Clinic, Kolkata, India
}

Background and Aim: Streptococcus pneumoniae constitutes one of the main causes of sepsis, bacteremia and meningitis and pneumonia in infants and small children. The study was aimed to determine the prevalence of these invasive diseases due to $S$. pneumoniae along with the serotype distribution in Eastern India.

Methods: During July 2012 to August 2013,104 children under 5 years were enrolled across 4 centres in eastern India presenting with pneumonia, bacteremia, meningitis. The blood culture samples were collected after written informed consent from parents. The cultures positive for pneumococcus were serotyped and their antimicrobial resistance was determined.

Results: Out of the 104 subjects $8.6 \%$ were culture positive and except one with Neissema meningitidis all were S. pneumoniae. Bacteremia was the commonest presentation amongst the culture positive cases with nearly $63 \%$, followed by pneumonia and meningitis. Of the serotypes identified $50 \%$ were common types as prevailing in the other centres as19A, 3, 6A and 19F. Rest $50 \%$ were uncommon like 35B, 38, 2, 18B. Most of the common serotypes presented with bacteremia in nephrotic syndrome cases.

The serotypes isolated are resistant to co-trimoxazole ( $80 \%$ cases) and erythromycin .None of the subjects were vaccinated and $90 \%$ of culture positive subjects were antibiotic naive. All subjects recovered during the hospital stay.

Conclusion: This study shows that pneumococcus was the commonest bacteria isolated in the studied population with bacteremia as the common presenting feature. Epidemiological surveillance system needs to be improved to get a better yield.

Acknowledgements: Dr Anand Manoharan and ASIP team

Conflict of interest

ISPPD-0139

Pneumococcal Pneumonia---Risky Business

\section{ATTITUDES AND KNOWLEDGE OF INTERNISTS AND CARDIOLOGISTS PHYSICIANS TO PNEUMOCOCCAL VACCINATION}

D. Curcio ${ }^{1}$, P. Valdez ${ }^{2}$, E. Zaidel' ${ }^{3}$ M.G. Abalos ${ }^{4}$, A. Cane ${ }^{4}$

${ }^{1}$ Infectious Diseases, Hospital Municipal Chivilcoy, Buenos Aires, Argentina; ${ }^{2}$ Internal Medicine, Hospital Velez Sarsfield, Buenos Aires, Argentina;

${ }^{3}$ Conarec, Buenos Aires, Argentina; ${ }^{4}$ Pfizer, Buenos Aires, Argentina

Background and Aims: Streptococcus pneumoniae infections are associated with high rates of morbidity and mortality for certain high-risk groups including adults $\geq 65$ years. The study aims to evaluate and compare attitudes and knowledge of cardiologists (CAR) and internists (INT) physicians about pneumococcal vaccination (PV), as well as the recommendations, administration practices and perceived barriers to vaccine delivery.

Methods :Questionnaires (available to view online) were administered by e-mail to 742 physicians. Assessment PV practices and attitudes were measured by the proportion test.

Results: Of 262 respondents included in the study (total response rate was 35\%), 135 (51.5\%) were INT, and $127(48.5 \%)$ were CAR. Ninety seven percent of CAR were aged $<35$ years compared to $70 \%$ of the INT, ( $p=0.0000$ [CI95\%;0.35551-0.18890]). Of all the respondents, 66\% (174/262) (46\% CAR and 86\% INT; $p=$ $0.0000[\mathrm{Cl} 95 \% ; 0.29794-0.50719])$ usually administer PV to at least some of their patients. The respondents most commonly indicate PV in persons with chronic respiratory diseases (81\% CAR and 75\% INT; $p=0.9363$ [CI95\%;0.09068-0.09827]), chronic cardiovascular disease (75\% CAR and 77\% INT; $p=0.9983$ [CI95\%;-0.09712-0.11217]) and those with inmmunosupresion condition (79\% CAR and 75\% INT; $p=0.5451$ [CI95\%;-0.14165-0.06314]. However, the proportion of respondents who usually prescribe PV was often significantly lower: $36 \%$ CAR vs $46 \%$ INT, $p=$ 0.1233(CI95\%;-0.22005-0.01778). Among CAR and INT, potential barriers reported for PV included the vaccine cost (40\% vs $41 \%, p=0.9702$ [CI95\%;-0.11390-0.12463]), patient concerns about vaccine safety $(27 \%$ vs $17 \%, p$ $=0.0786[\mathrm{Cl} 95 \% ;-0.03154-0.19240])$, and inadequate reimbursement $(52 \%$ vs $75 \%, p=0.6469[\mathrm{Cl} 95 \% ;-0.19731-$ $0.00262])$.

Conclusion: A low percentage of CAR and INT recommend PV to their patients, without significant differences related to their attitudes and knowledge. Because our respondents usually attend patients with risk factors por pneumococcal disease, the commitment of these specialists with the PV is crucial.

No conflict of interest 
ISPPD-0138

Pneumococcal Pneumonia---Risky Business

\title{
RISK FACTORS FOR PNEUMOCOCCAL INFECTION IN INTENSIVE CARE PATIENTS: A MISSED OPPORTUNITY FOR VACCINATION
}

\author{
D. Curcio ${ }^{1}$, M.G. Abalos ${ }^{2}$, A. Cane ${ }^{2}$, The Latin American Antibiotic Use in Intensive Care Unit Group ${ }^{3}$ \\ ${ }^{1}$ Infectious Diseases, Hospital Municipal Chivilcoy, Buenos Aires, Argentina; ${ }^{2}$., Pfizer, Buenos Aires, Argentina; ${ }^{3}$., ., ., Argentina
}

Background and Aims: Hospitalized patients in intensive care unit (ICU) are frequently at risk for subsequent pneumococcal disease (PD). Despite this risk, they are often not vaccinated in this setting. This study assessed the characteristics of patients in ICU in relation to their candidacy for pneumococcal vaccination (PV).

Methods: This was an observational, cross-sectional study in which 22 Latin America (LA) ICUs completed a webbased data collection form with data from the hospitalized adults ( $>18 \mathrm{y}$ ) patients (a one-day point prevalence study). The main outcome measures were documentation by chart review of risk factors (RF) for PD, communityacquired pneumonia (CAP) and PV status.

Results: Of the 178 patients, $128(72 \%)$ had indication for pneumococcal vaccine according to the Centers for Disease Control's Advisory Committee on Immunization Practices (ACIP) recommendation. Despite this only 5/128 patients (4\%) had immunization status documented The mean number of RF for PD was $1.6(95 \% \mathrm{Cl} ; 1.4-1.6)$, with the majority of patients being over age $65(40 \%)$, having chronic heart or lung disease (40\% and $26.5 \%$ respectively) and diabetes (37.5\%). High risk conditions for PD were observed in 31 (24\%) patients. Sixty-three patients (35\%) were admitted in ICU due to CAP. Among these patients rate of PV was $0 \%$ and the mean of RF $2(95 \% \mathrm{Cl} ; 1.7-2.4 ; p=$ $0.2400)$.

Conclusion: Most patients admitted to the LA ICU had multiple indications for PV. However, only a small percentage had received vaccination previous their hospital admission. Because hospitalization provides an opportunity to vaccinate patients at high risk for developing serious PD complications, eligibility screening and administration of the PV prior to discharge in qualified ICU patients may improve the immunization rates.

No conflict of interest

ISPPD-0335

Pneumococcal Pneumonia---Risky Business

\section{IMPACT OF TWO PNEUMOCOCCAL CONJUGATE VACCINES ON HOSPITALIZATIONS FOR LOWER-TRACT RESPIRATORY INFECTIONS (LTRI) IN QUEBEC, CANADA}

\author{
Z. Zhou ${ }^{1}$, G. Deceuninck ${ }^{1}$, F. Boucher ${ }^{2}$, R. Gilca ${ }^{3}$, P. De Wals ${ }^{4}$ \\ ${ }^{1}$ Vaccine Research Unit, Quebec University Hospital Research Centre, Quebec City, Canada; ${ }^{2}$ Pediatrics, Quebec University Hospital Research Cen- \\ tre, Quebec City, Canada; ${ }^{3}$ DRBO, Quebec National Public Health Institute, Quebec City, Canada; ${ }^{4}$ Social and Preventive Medicine, Universi- \\ té Laval, Quebec City, Canada
}

Background: In Quebec, Canada, a pneumococcal conjugate vaccine (PCV) program was implemented in December 2004. PCV7 was first used and replaced by PCV-10 in June 2009. From the beginning, vaccine uptake has been high: $>90 \%$ of children receiving the recommended number of doses. Our aim was to assess the relative impact of these two vaccines on hospitalizations for lower respiratory tract infection (LTRI).

Methods: The study population included children born in 2007-2010 and observed up to their second year anniversary. Hospitalization records with a main diagnosis of LTRI were identified in the provincial administrative database MedEcho. Monthly birth numbers were provided by the Quebec Statistics Institute. Birth cohorts were classified according to the main vaccine used ( $\geq 90 \%$ of doses). Cumulative hospitalization frequencies (CHF) from 3 to 23 months of age were computed. Multivariate Poisson models adjusting for age, month of birth, virus circulation and ambient temperature were used to assess the vaccine program effect on first hospitalization.

Results: The analysis included 9,120 hospitalizations for LTRI among children with complete follow-up. CHF was 3.35 $\%$ among children exposed to PCV-7 and 3.11 in those exposed to PCV10 ( $p=0.07)$. Multivariate analyses showed a statistically significant effect of age, month of birth, ambient temperature, respiratory syncytial virus (RSV), influenza and metapneumovirus circulation but not of different vaccines.

Conclusion: Results of ecological studies should be interpreted with care but we were not able to demonstrate any substantial difference between PCV7 and PCV10 on hospitalizations for LTRI.

Conflict of interest 
ISPPD-0145

Pneumococcal Pneumonia---Risky Business

\title{
REVIEW OF REAL WORLD EFFECTIVENESS IN ALL-CAUSE PNEUMONIA FOLLOWING EXPANDED USE OF PNEUMOCOCCAL CONJUGATE VACCINES
}

\author{
C. Roberts ${ }^{1}$, R. Farkouh ${ }^{1}$, R. Klok ${ }^{2}$, D. Strutton ${ }^{1}$ \\ ${ }^{1}$ Vaccines, Pfizer, Collegeville, USA; ${ }^{2}$ Vaccines, Pfizer, Den Hague, Netherlands
}

Background: The 7-valent pneumococcal conjugate vaccine (PCV7) has consistently demonstrated reductions against all-cause pneumonia across countries. We compare methods and results of real world pneumonia effectiveness studies of PCV immunization programs.

Methods: To identify real world data on effectiveness of pediatric PCVs in immunization programs against all-cause pneumonia, we searched PubMed for articles published January 1, 2002 to September 1, 2013. Search terms included 'pneumonia, incidence, increase, decrease, reduction, and PCV'. Data extracted included country, data source, vaccine, schedule, age groups, pre and post period, pneumonia definition, and vaccine effectiveness (VE). Articles reporting multiple regions were separately analyzed. VE estimates were those reported by the authors, or percent change in incidence if not specified.

Results: From 449 titles 29 articles, reporting 35 regional analyses, were identified. Among these, 28 articles reported hospitalized estimates and 6 reported outpatient estimates. Cases were identified by ICD-9/10 coding ( $n$ $=24$ ) or chart review $(n=5)$, with 3 applying WHO chest radiograph criteria. PCV7 effectiveness was reported in 26 articles, PCV7/13 in 2, and PCV10 in 1. Median PCV7 and PCV7/13 VE estimates were $24 \%$ in children $<1$ year $(n=6$, IQR=22\%-30\%), 38\% in children $<2$ years ( $n=23$, IQR $28 \%-52 \%)$, and $44 \%$ in children $<5$ years $(n=4$, IQR $=35 \%-47 \%)$. The PCV10 study examined 5 regions in children 2-24 months, with median reduction of $23 \%$ (IQR $2 \%-27 \%$ ). Conclusion: PCV7 and PCV7/13 consistently show significant all-cause pneumonia effectiveness in vaccinated age groups. Study designs vary by pneumonia definition and pre-/post-period duration. Researchers should cautiously compare across pneumonia effectiveness studies.

Conflict of interest

ISPPD-0499

Pneumococcal Pneumonia---Risky Business

\section{EVALUATION OF RESPIRATORY VIRAL CO-INFECTION WITH MICROBIOLOGICALLY CONFIRMED PNEUMOCOCCAL PNEUMONIA (MCPP) IN THE PERCH STUDY}

The PERCH Study Group ${ }^{1}$

${ }^{1}$ International Vaccine Access Center, The Johns Hopkins School of Public Health, Baltimore MD, USA

Background: There are limited data on the role of respiratory viral co-infection among children with microbiologically confirmed pneumococcal pneumonia (MCPP).

Methods: In cases aged 28 days - 59 months hospitalized with WHO-defined severe/very severe pneumonia and age frequency-matched community controls in 7 Africa and Asia sites, induced sputum (cases only) and nasopharyngeal swabs were tested for 18 viruses by Fastrack PCR. MCPP was defined as Streptococcus pneumoniae cultured from a sterile specimen or lytA+ PCR from lung or pleural aspirate. Pneumococcal colonization was defined as $L y t A+$ in the nasopharynx. We compared the prevalence of viruses in the nasopharynx between MCPP and nonMCPP cases in the 5 African sites with MCPP cases.

Results: Unadjusted prevalences are shown in the Figure. After adjusting for age and site, no comparison was significant. Of seven MCPP cases with pneumococcus from lung aspirates, two had viruses in the lung (coronavirus 43 and cytomegalovirus). $72.2 \%$ of all cases had pneumococcal nasopharyngeal colonization. There was no difference in the mean number of viruses among colonized vs. not colonized with pneumococcus: 1.77 vs 1.53 among cases and 1.36 vs 1.12 among controls.

Conclusion: MCPP cases did not have an increased frequency of viral co-infection compared with non-MCPP cases. The few number of MCPP cases limited analyses. The relationship between pathogen co-infection in the nasopharynx and lung needs further study.

Figure: Prevalence of viruses in the nasopharynx among MCPP and non-MCPP cases. 


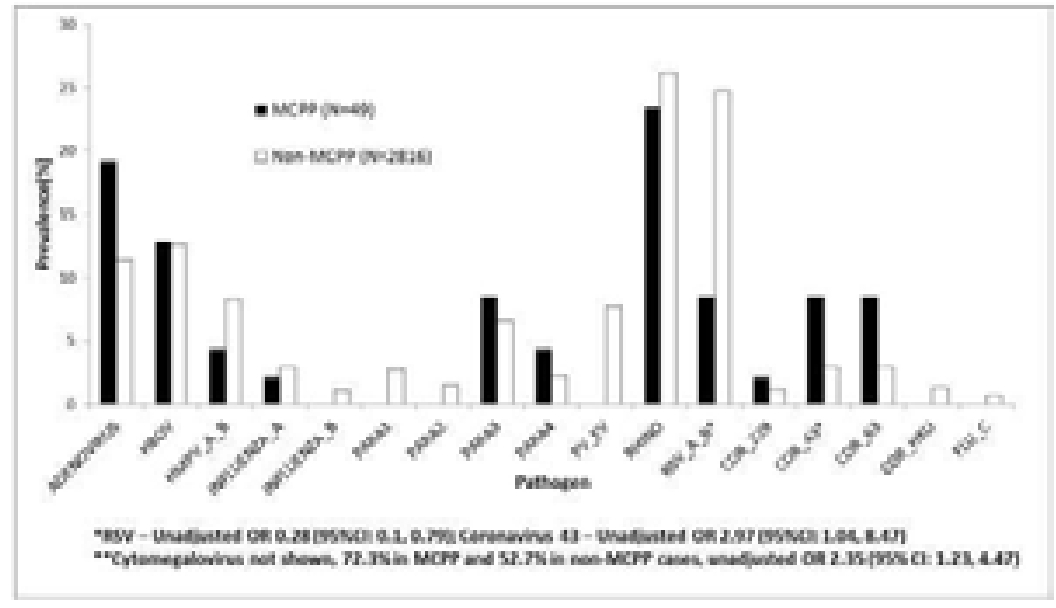

No conflict of interest

ISPPD-0472

Pneumococcal Pneumonia---Risky Business

\title{
CONTRIBUTION OF STREPTOCOCCUS PNEUMONIAE TO THE BURDEN OF COMMUNITY ACQUIRED PNEUMONIA IN HOSPITALIZED CHILDREN IN VENEZUELA
}

\author{
K. Gomez-Castellano ${ }^{1}$, I.A. Rivera-Olivero' ${ }^{1}$, N.V. Ortiz ${ }^{2}$, P. Vigilanza ${ }^{2}$, J.H. De Waard ${ }^{3}$
}

${ }^{1}$ Lab. Tuberculosis, Instituto de Biomedicina, Caracas, Venezuela; ${ }^{2}$ Departamento de Pediatria, Hospital de Niños JM de los Rios, Caracas, Venezuela; ${ }^{3}$ Lab Tuberculosis, Instituto de Biomedicina, Caracas, Venezuela

Background: Community-acquired pneumonia (CAP) is a serious cause of morbidity among children in developed countries. Hospitalization for CAP is common but often no etiological agent can be established. Here, we estimate the contribution of Streptococcus pneumoniae to CAP in children.

Method: A total of 72 children under 5 year old with CAP admitted to Hospital de niños "JM de los Rios", were studied prospectively from June 2010 through March 2012. Tests for S. pneumoniae included blood culture, blood PCR for lytA and cpsA, urinary pneumococcal antigen detection and for pleural fluid (when available) culture and PCR. Additionally, nasopharyngeal swabs were collected for bacteriologic culture to evaluate carriage of $S$. pneumoniae.

Results: All 72 blood cultures were negative for S. pneumoniae. PCR on blood was positive in 12.5\% (9/72). Urine antigen testing was positive in $52.7 \%(38 / 72)$ of the children. Two out of 3 pleural fluids tested positive for $S$. pneumoniae with culture and/or PCR. S. pneumoniae carriage rate was $56 \%$, and the most frequent serotypes isolated were $14,19 \mathrm{~F}$ and $656.9 \%(41 / 72)$ of the children were positive for $1 \mathrm{~S}$. pneumoniae test (PCR, urine antigen, carrier or culture positive) and $29.16 \%(21 / 72)$ had 2 or more positive test results.

Conclusion: Addition of techniques increased the diagnosis of a presumptive pneumococcal etiology. Preliminary results from this study suggest that the current contribution of S. pneumoniae to CAP is between 30 and $56 \%$; much higher than previously reported utilizing standard laboratory techniques in Venezuela.

No conflict of interest

ISPPD-0475

Pneumococcal Pneumonia---Risky Business

\section{AETIOLOGY OF PNEUMONIA AMONG PATIENTS AGED 5 YEARS AND OVER IN RURAL GAMBIA}

E.W. Green ${ }^{1}$, U.N. Ikumapayi ${ }^{2}$, U. Uchendu ${ }^{2}$, D. Ameh², M. Ndiaye ${ }^{2}$, H. Badji' ${ }^{2}$ A. Manjang ${ }^{2}$, D. Nsekpong ${ }^{2}$, S. Jarju ${ }^{2}$,

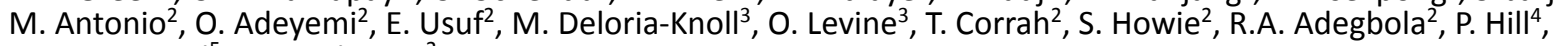
B. Greenwood ${ }^{5}$, G. Mackenzie ${ }^{2}$

${ }^{1}$ Clinical Services Department, Medical Research Council: The Gambia Unit, Fajara, Gambia; ${ }^{2}$ Pneumococcal Surveillance Project, Medical Research Council: The Gambia Unit, Fajara, Gambia; ${ }^{3}$ International Health, Johns Hopkins Bloomberg School of Public Health, Baltimore, USA ${ }^{4}$ Centre for International Health, University of Otago, Dunedin, New Zealand; ${ }^{5}$ Department of Disease Control, London School of Hygiene \& Tropical Medicine, London, United Kingdom

Background and Aims: We describe the aetiology of pneumonia among patients aged $\geq 5$ years in a rural area of The Gambia.

Methods: We conducted prospective pneumonia surveillance from May 2008 until August 2013 in six health facilities in the Basse Health and Demographic Surveillance System in eastern Gambia. PCV7 was introduced in August 2009, PCV13 in May 2011. All patients were screened by nurses for referral to a clinician who used standard criteria to diagnose pneumonia. Blood cultures were collected from every patient and a subset of patients had a lung or pleural aspirate collected. Samples were processed using conventional methods.

Results: Over 64 months of surveillance, 1224 specimens were collected from 961 patients with suspected pneumonia (age 5-9 $n=396,10-14 n=121,15-49 n=319, \geq 50 n=125$ ). There were 109 positive specimens from 92 patients ( 78 blood cultures, 30 lung aspirates and one pleural aspirate). 60 patients had Streptococcus 
pneumoniae isolated of which 32 were serotype 1,15 serotype 5 , and a total of 50 were PCV13 serotypes. Other causative organisms were Staphylococcus aureus $(n=10)$ and Salmonella spp $(n=7)$. The case-fatality rate was $1 \%$ for patients aged $<50$ and $3 \%$ for patients aged $\geq 50$.

Conclusion: Among patients aged $\geq 5$ years with suspected pneumonia, S. pneumoniae was the predominant organism and PCV13 serotypes accounted for over $80 \%$ of pneumoccal isolates. If the introduction of PCV13 is associated with herd protection in older age groups there is the potential for a reduction in pneumococcal pneumonia in adults.

No conflict of interest

ISPPD-0485

Pneumococcal Pneumonia---Risky Business

\section{ELEVATED CRP IS ASSOCIATED WITH BACTERIAL PNEUMONIA IN CHILDREN, BUT MAY NOT DISTINGUISH PNEUMOCOCCAL FROM OTHER BACTERIAL PNEUMONIAS: PERCH STUDY}

The PERCH Study Group ${ }^{1}$

${ }^{1}$ International Vaccine Access Center, The Johns Hopkins Bloomberg School of Public Health, Baltimore, USA

Background: C-reactive protein (CRP) is an acute-phase reactant associated with bacterial infection and may help in distinguishing pneumococcal pneumonia.

Methods: In the Pneumonia Etiology Research for Child Health (PERCH) study in South Africa, blood CRP levels and whole blood lytA were tested in 775 cases age 28 days - 59 months hospitalized with WHO-defined severe or very severe pneumonia and in 177 age frequency-matched community controls, including $25 \%$ with upper-respiratory tract infection and $45 \%$ with blood lytA positivity. Nasopharyngeal (NP) swabs and induced sputum (IS) were also obtained from cases for culture and multiplex-PCR for 33 respiratory pathogens. Odds ratios (OR) for the proportion with $\mathrm{CPR} \geq 40 \mathrm{mg} / \mathrm{L}$, a previously established threshold for pneumococcal pneumonia, for various pneumonia outcomes were calculated.

Results: All 3 cases with pneumococcus detected in pleural fluid or blood culture had CRP $\geq 40 \mathrm{mg} / \mathrm{L}$. CRP was associated with other markers of pneumococcal and bacteremic infection (Figure). A positive association with influenza and negative association for respiratory syncytial virus associated pneumonia remained after adjusting for pneumococcal and all-bacterial infection in NP and/or IS.

Figure: Percent with CRP $\geq 40 \mathrm{mg} / \mathrm{L}$ by outcome

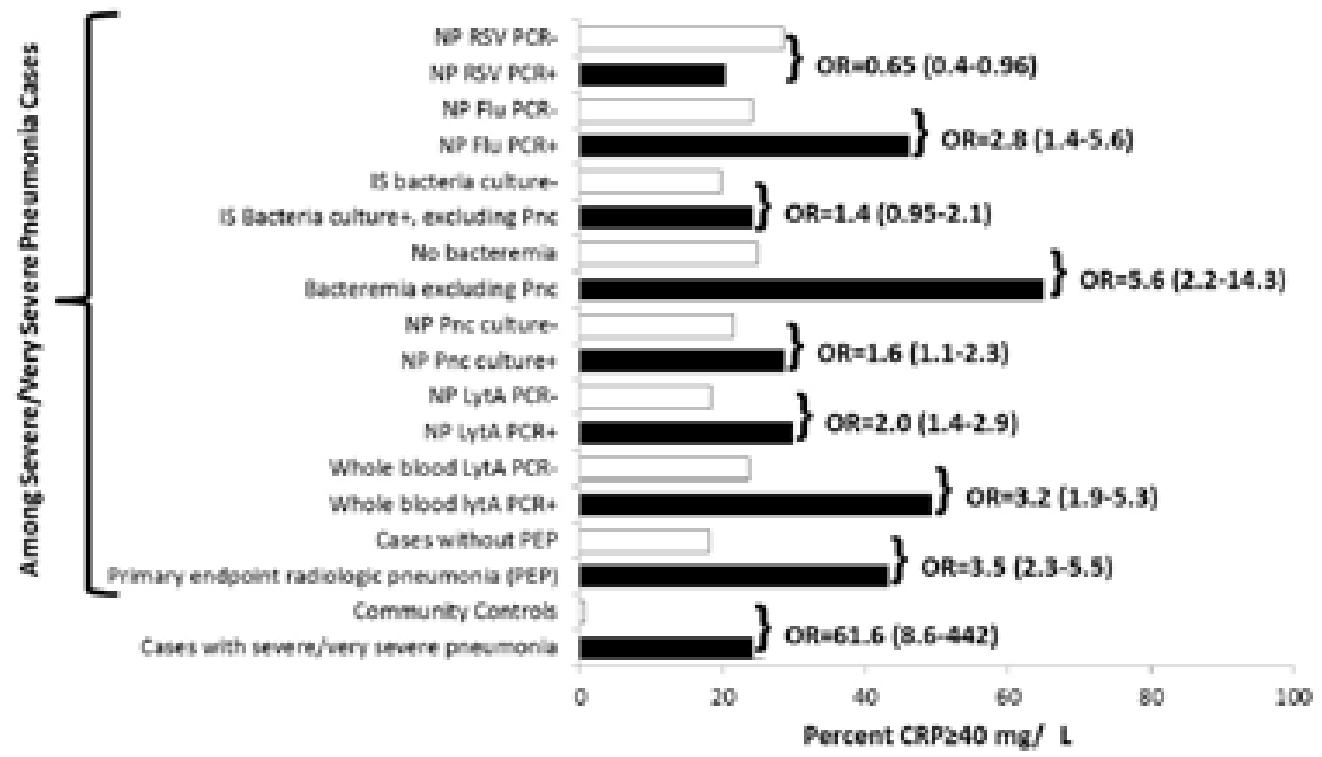

Conclusion: Elevated CRP is suggestive of bacterial etiology in children with WHO-defined severe or very severe pneumonia, but may not distinguish pneumococcus from other bacterial pathogens. The association between viral infection and CRP is potentially confounded by the low sensitivity of blood cultures identifying bacterial infection.

No conflict of interest 
ISPPD-0119

Pneumococcal Pneumonia---Risky Business

\title{
STUDY OF RISK FACTORS WITH TREATMENT OUTCOME IN HOSPITALIZED COMMUNITY ACQUIRED PNEUMONIA
}

K.R. Raveendra ${ }^{1}$, K.G. Prakash ${ }^{1}$, J. Jayachandra ${ }^{1}$

${ }^{1}$ Internal medicine, Bangalore Medical College Victoria hospital, Bangalore, India

Introduction: Presence of risk factors in community acquired pneumonia (CAP) patients plays an important role in the diagnosis and management. It's really a challenge to treat CAP patients with multiple risk factors as it has a direct impact on its mortality and morbidity.

Aim of the Study:

1. To study the risk factor profile in hospitalized CAP patients at a tertiary hospital.

2. To study the impact of risk factors on the treatment outcome in these cases.

Materials and Methods: 450 hospitalized CAP patients' hospital records were studied and analyzed for the various risk factors and treatment outcome for a period of one year between 1st January 2012 - 31sr December 2012. The risk factors profile was correlated to various parameters which include recovery, prolonged stay in the hospital, unresolved pneumonia and deaths.

Results: Of 450 patients, 267 were males and 183 females. 218 patients belonged to the age group of $41-60$ years. 180 patients did not have any risk factors. 208 patients had $1-2$ risk factors and 62 patients had $\geq 3$ risk factors. Commonest risk factors were smoking (93), alcohol (66), diabetes (45), COPD (27), hypertension (27) and immunosuppression (15). All adverse parameters namely prolonged hospital stay, unresolved pneumonia, discharge against medical advice (DAMA) and deaths were noted in 10/16, 15/30,12/24 and 5/11 in patients with $\geq 3$ risk factors respectively.

Conclusion: Risk factors identification and treatment at the earliest along with proper management of neumonia will decrease the mortality and morbidity of hospitalized CAP patients

No conflict of interest

ISPPD-0134

Pneumococcal Pneumonia---Risky Business

\section{DISEASE BURDEN OF PNEUMONIA IN KOREAN ADULTS AGED OVER 50 YEARS STRATIFIED BY UNDERLYING COMORBIDITIES}

\author{
K.S. Jung ${ }^{1}$, C.G. Yoo ${ }^{2}$, H.J. Kim ${ }^{3}$
}

${ }^{1}$ Internal Medicine, Hallym University Sacred Heart Hospital, Anyang, Korea; ${ }^{2}$ Internal Medicine, Seoul National University College of Medicine, Seoul, Korea; ${ }^{3}$ Market Access Department, Pfizer Pharmaceuticals Korea Ltd, Seoul, Korea

Background and Aims: Community-acquired pneumonia (CAP) shows high mortality rate despite antibiotics treatment but there are no sufficient data on disease burden of pneumonia in Korea. This study was conducted to assess disease burden of pneumonia in adults according to underlying diseases types and age groups.

Methods: We performed a multi-center, retrospective, observational study and collected data targeting for CAP patients ( $\geq 50 \mathrm{yr}$ ) from 11 hospitals. Data were assessed by retrospective review of patient medical records. Disease burden was defined as measured by total per-capita medical fee, severity, hospital length of stay (LOS), and mortality. Medical fee was calculated by multiplying the total use of the medical resource item during the hospital LOS with the medical cost unit price for the item. (1 USD =1,100 Korean Won)

Results: A total 693 patients were included and $83.4 \%$ had the underlying disease. Total per-capita medical fees were the highest and the hospital LOS was the longest when the patients had chronic renal disease (USD $2,060 \pm 1,309,10.72$ days). If the patients had CNS disorder, the severity level by CURB 65 score and the mortality rate were the highest $(1.88,9.4 \%)$. The disease burden was higher markedly with advancing age. Also, having any kinds of underlying disease with pneumonia was significantly contributed to the burden of disease.

Conclusion: In Korea, the appropriate prevention of pneumonia should be considered as effective strategy, especially the patients with having underlying disease or older age group.

No conflict of interest

ISPPD-0073

Pneumococcal Pneumonia---Risky Business

\section{INVASIVE PNEUMOCOCCAL DISEASE, CO-MORBIDITIES AND POLYSACCHARIDE VACCINE USE IN 5-15 YEAR-OLD CHILDREN IN ENGLAND AND WALES}

\author{
S. Ladhani ${ }^{1}$, N.J. Andrews ${ }^{1}$, P. Kaye ${ }^{1}$, R. Borrow ${ }^{2}$, M.P.E. Slack ${ }^{3}$, E. Miller ${ }^{1}$ \\ ${ }^{1}$ Immunisation Department, Public Health England, London, United Kingdom; ${ }^{2}$ Vaccine Evaluation Unit, Public Health England, Manchester, Unit- \\ ed Kingdom; ${ }^{3}$ Respiratory and Vaccine Preventable Bacteria Department, Public Health England, London, United Kingdom
}

Background: In the United Kingdom, at-risk children aged $\geq 2$ years are recommended the 23-valent pneumococcal polysaccharide vaccine (PPV23) in addition to routine pneumococcal conjugate vaccination. This study describes the epidemiology, serotype distribution, clinical characteristics, vaccination status and reasons for non-vaccination in 5-15 year-old children with invasive pneumococcal disease (IPD). 
Methods: Public Health England conducts enhanced national surveillance of IPD in England and Wales. In 2012, general practitioners (GPs) were contacted to complete a questionnaire for 5-15 year-old children with laboratoryconfirmed IPD diagnosed during two epidemiological years, July 2009-June 2011.

Results: During 2009-11, 447 IPD episodes occurred in 439 children (incidence, 2.2/100,000) and GPs of 423/439 (96.4\%) cases completed the questionnaire. Co-morbidity was reported in 124 (29.3\%); a third each were immunocompromised, had chronic respiratory disease or other co-morbiditieS. pneumonia was the most common presentation (332/439, 75.6\%) and IPD-related case-fatality was 1.8\% (8/439). Only 26.6\% (33/124) children with co-morbidities had received PPV23 and development of PPV23-IPD was not associated with prior PPV23 vaccination (aOR 1.09; 95\%Cl, 0.36-3.32; $p=0.88$ ), even when analysis was restricted to the extra 11 PPV23 serotypes not contained in the 13-valent pneumococcal conjugate vaccine (aOR 1.70; $95 \% \mathrm{Cl}, 0.30-9.76 ; p=0.55$ ). GPs of eligible but unvaccinated cases with co-morbidities were mostly unaware that the child required PPV23 and/or expected paediatricians to inform them to administer the vaccine.

Conclusion: Only a quarter of children with co-morbidities who developed IPD had received PPV23 prior to infection. Among PPV23-vaccinated children with co-morbidities, however, there was no evidence of protection against PPV23 serotypes.

No conflict of interest

ISPPD-0109

Pneumococcal Pneumonia---Risky Business

\section{PRELIMINARY RESULTS OF A PROSPECTIVE POPULATION-BASED SURVEILLANCE: BURDEN OF COMMUNITY- ACQUIRED PNEUMONIA IN ADULTS, IN SOUTH CONE OF LATIN-AMERICA}

G. Lopardo ${ }^{1}$, D. Fridman ${ }^{1}$, E. Raimondo ${ }^{2}$, H. Bagnulo ${ }^{3}$, H. Albornoz ${ }^{4}$, D. Goleniuk ${ }^{5}$, M. Sanabria ${ }^{6}$, E. Baez ${ }^{7}$, D. Stamboulian $^{1}$

${ }^{1}$ Research Department, Fundación Centro de Estudios Infectológicos (Funcei ), Ciudad Autónoma de Buenos Aires, Argentina; ${ }^{2}$ Infectious Department, Hospital F. López Lima, General Roca Río Negro, Argentina; ${ }^{3} \mathrm{CTI}$, Hospital Maciel, Montevideo, Uruguay; ${ }^{4}$ Infectious Department, Sanatorio Casmer, Rivera, Uruguay; ${ }^{5} \mathrm{CTI}$, Sanatorio Casmer, Rivera, Uruguay; ${ }^{6}$ Intensive Care Unit, Hospital Regional de Concepción. Instituto de Previsión Social, Concepción, Paraguay; ${ }^{7}$ Infectious Department, Centro Médico La Costa y Servicios Médicos Migone, Asunción, Paraguay

Background: Community-acquired pneumonia (CAP) is one of the leading causes of death. The incidence of CAP in Latin-America is unknown.

Methods: Subjects residing in Roca city (Argentina), Rivera city (Uruguay) and Concepción city (Paraguay) diagnosed with radiological confirmed CAP were included. Incidence rate was defined as the number of cases of CAP in the population older than 18 years residing in the surveillance area, divided by the total population at risk.

Results: From January 2012 to August 2013, 2893 subjects were assessed, and 1337 were diagnosed with CAP. Global incidence in Roca was 8.4 (CI95\% 2.4-14.4) per 1000 population and 34 for those $>65$ y (15.5-52.5); in Rivera, $6.4(\mathrm{Cl} 0.6-13.4)$ and $17.4(\mathrm{Cl} 0-34.4)$ and in Concepción, $3(\mathrm{Cl} 2.99-11.4)$ and $14(\mathrm{Cl} 0-39)$, respectively. Mean age: 62.7 years; female, $55.2 \%$. 64\% of episodes were hospitalized; duration of stay (DS) 6 days (DS 4-9); loss of activities associated with CAP: 10 days (DS 7-15). Mortality rate was $11,06 \%$; in patients $>65 \mathrm{y}, 17,31 \%$. $17 \%$ of subjects had ever received pneumococcal vaccine; mortality in vaccinated subjects was $22 \%$ and in those never vaccinated, $9 \%$ ( $p$ $=0.2$ ). Mortality in subjects with positive urinary antigen for $S$. pneumonia was $18,6 \%$ vs. $10,7 \%$ in subjects with a negative result $(p=0.03)$.

Conclusion: the incidence in adults, particularly $>65$ y was substantial. Mortality in CAP-episodes with a positive urinary antigen was significantly higher. Pneumococcal vaccination was not associated with lower mortality.

No conflict of interest

ISPPD-0422

Pneumococcal Pneumonia---Risky Business

\section{CASE-CONTROL STUDY ON EFFECTIVENESS OF PNEUMOCOCCAL CONJUGATE VACCINE (PCV) AGAINST PRESUMED BACTERIAL PNEUMONIA (PBP) HOSPITALIZATION IN HIV-UNINFECTED SOUTH AFRICAN CHILDREN}

S.A. Madhi' ${ }^{1}$, M.J. Groome ${ }^{2}$, H. Zar ${ }^{3}$, C. Kopongo ${ }^{4}$, C. Mulligan ${ }^{3}$, S. Nzenze ${ }^{2}$, D. Moore ${ }^{2}$, E. Zell ${ }^{5}$, C. Whitney ${ }^{6}$

${ }^{1}$ Center for Respiratory and Meningitis Diseases, National Institute for Communicable Diseases, Sandringham Johannesburg, South Africa; ${ }^{2}$ MRC: Respiratory and Meningeal Pathogens Research Unit, University of the Witwatersrand, Bertsham Johannesburg, South Africa; ${ }^{3}$ Red Cross Hospital Paediatrics, University of Cape Town, Cape Town, South Africa; ${ }^{4}$ Paediatric Department, Ngwelezane Hospital, Richards Bay, South Africa; ${ }^{5}$ Respiratory Diseases Branch, Center for Diseases Control and Prevention, Atlanta USA, South Africa; ${ }^{6}$ Respiratory Diseases Branch, Center for Diseases Control and Prevention, Atlanta USA, South Africa

Background: 7-valent PCV was introduced in South Africa in April 2009 using a 2+1 schedule (doses at age 6, 14 and 39 weeks), superseded with 13-valent PCV in May 2011. We evaluated PCV effectiveness against hospitalization for PBP in HIV-uninfected children.

Methods: A matched case-control study was undertaken at three sites between April 2009 and July 2012. Presumed bacterial oneumonia (PBP) cases had either WHO-defined radiographically-confirmed pneumonia or 'other infiltrate' on chest radiograph with C-reactive protein $\geq 40 \mathrm{mg} / \mathrm{l}$. Hospitalized controls (HC) were matched to cases by age, site and HIV-infection status. Age-matched community controls (CC) identified through birth registries were enrolled from Soweto; CC known to be HIV-infected were excluded. Vaccine effectiveness estimates were adjusted (aVE) for potential confounders.

Results: Of 1,449 HIV-uninfected enrolled PBP cases, 1,318 had $\geq 1$ matched HC ( $n=1,997) ; 894$ PBP cases in Soweto 
had $\geq 1 \mathrm{CC}(n=2,662)$. Among children aged $\geq 8$ weeks and using HC, aVE for age-appropriate PCV schedule was $22.4 \%$ (95\% Cl: $-6.2,43.3 \%)$ overall, 39.4\% (95\% Cl: 9.4, 59.5\%) in Soweto. Among children 16-103 weeks and using HC, aVE for age-appropriate PCV was $38.3 \%(95 \% \mathrm{Cl}: 6.6,59.2 \%)$, overall, $54.9 \%(95 \% \mathrm{Cl}: 31.4,70.3 \%)$ in Soweto. In Soweto, aVE using CC was similar to that observed with $\mathrm{HC}$, i.e. $33.2 \%(95 \% \mathrm{Cl}: 6.2,52.4 \%)$ and $52.3 \%(95 \% \mathrm{Cl}: 25.2$, $69.6 \%$ ) in age-groups $\geq 8$ weeks and $16-103$ weeks, respectively.

Conclusion: PCV effectiveness against PBP in our study was similar to efficacy measured by randomized controlled trials using different vaccination schedules. Two different control types (hospital and community) yielded similar results in the Soweto context.

Conflict of interest

ISPPD-0554

Pneumococcal Pneumonia---Risky Business

\title{
AN INCREASED INCIDENCE OF PNEUMOCOCCAL PNEUMONIA AFTER THE GREAT TOHOKU EARTHQUAKE AND TSUNAMI: DISASTER IMPACT AMPLIFIED WITH PNEUMOCOCCUS INFECTION
}

T. Makino ${ }^{1}$, T. Aoyagi ${ }^{2}$, H. Takeda ${ }^{3}$, H. Kunishima ${ }^{4}$, M. Kaku² $^{2}$, K. Oishi $^{1}$

${ }^{1}$ Infectious Diseases Surveillance Center, National Institute of Infectious Diseases, Tokyo, Japan; ${ }^{2}$ Graduate School of Medicine, Tohoku University, Sendai, Japan; ${ }^{3}$ Internal Medicine Department, Saiseikai Yamagata Saisei Hospital, Yamagata, Japan; ${ }^{4}$ General Internal Medicine, Saint Marianna Medical School, Kawasaki, Japan

Background: The great East Japan Earthquake and Tsunami on March 11th 2011 devastated the broad area with the most aged population in Japan, followed by the increased incidence of pneumonia. We aimed to evaluate the health impact of pneumonia by Streptococcus pneumoniae, considering the opportunity of prevention by available vaccine.

Method: Retrospective population-based survey was conducted in the two prefectures: Miyagi (Tsunami affected) and Yamagata (Control). Key hospitals of these two prefectures reported the cases of pneumococcal pneumonia (PP) and invasive pneumococcal diseases (IPD) seen through two years between 1st November 2010 through 31th October 2012. Our analysis included patients' onset date and location of residence.

Result: Distribution of 1,159 registered cases revealed the sharp increase of PP cases in Miyagi immediately after the disaster for about three weeks. Even next winter Miyagi experienced higher incidence of PP. Collective population-based incident rate (per 100,000 person years) over the entire study period was statistically significantly higher in Miyagi (80.0) than in Yamagata (44.7) $(p=0.0001)$. Cases of PP in Miyagi were more likely to reside at the evacuation camp.

Conclusion: The natural disaster increased the incidence of PP among aged population. Possible reasons include deprivation of regular medical and nursing care, high density of population and insufficient living supply in evacuation shelters, and psychologically stress weakening immune system. Delayed recovery of infrastructures and cold climate in Tohoku might have even worsened the PP burden on senior population in the following winter.

No conflict of interest

ISPPD-0165

Pneumococcal Pneumonia---Risky Business

\section{INVASIVE PNEUMOCOCCAL DISEASE SEROTYPES AND PENICILLIN RESISTANCE AMONG UNDER-FIVE CHILDREN AND IMPLICATIONS TO CHOICE OF POLYSACCHARIDE CONJUGATE VACCINES FOR INDIA}

\author{
V. Manchanda ${ }^{1}$, P. Kinha ${ }^{1}$, H. Gautam ${ }^{1}$, A. Manoharan ${ }^{2}$ \\ ${ }^{1}$ Clinical Microbiology and Infectious Diseases, Chacha Nehru Bal Chikitsalaya, NEW DELHI, India; ${ }^{2}$ Benjamin M Pulimood Laboratories for Infec- \\ tion Immunity and Inflammation (BMPLIII) Department of Medicine Unit I \& Infectious Diseases, Christian Medical College, Vellore, India
}

Background and Aims: Developing nations have almost 2000 times' higher disease burden of IPD than that in the developed world. The present study aimed to determine occurrence of invasive pneumococcal disease in U-5 children. The study also intended to determine the prevalent serotypes and susceptibility profiles of Streptococcus pneumoniae in India.

Methods: The present study was an observational, prospective, hospital based study. Children aged 2 months-5 years with clinical syndromes including pneumonia with radiographic evidence or meeting WHO clinical criteria (WHO/ARI/90.5) or clinically suspected meningitis suggestive of bacterial meningitis were included. Blood, CSF and/ or pleural fluid were collected for culture. Bacterial identification and susceptibility testing was performed using Vitek2C system. Serotyping was performed using Quellung Testing.

Results: A total of 953 children were enrolled during September 2010 through March 2013. S. pneumoniae was isolated from 46 children. Culture positivity was higher in children -with pneumonia with effusion (23.6\%) followed -with meningitis (8.1\%). Penicillin resistance (24\%) was observed using meningitis breakpoints (CLSI). No penicillin resistance was observed using pneumonia breakpoints. Case fatality was $5.8 \%$ in study population and $17.3 \%$ in culture positive cases. Twenty six pneumococcal serotypes were implicated. Existing vaccine coverage was $28.26 \%$, $69.5 \%$ which can be increased to $71.7 \%, 78.3 \%$ and $84.8 \%$ for $7-, 10-$ and 13 -valent vaccine respectively using India specific conjugated pneumococcal vaccines.

Conclusion: IPD has significant morbidity and mortality in Indian setting. Penicillin still remains choice of treatment for pneumococcal pneumonia. However, meningitis cases should be treated with high dose 3rd generation cephalosporins. Existing pneumococcal vaccines have good coverage which can be further enhanced using India specific vaccine. 
ISPPD-0523

Pneumococcal Pneumonia---Risky Business

\title{
INVASIVE BACTERIAL DISEASE (IBD) IN CHILDREN <5 YEARS IN CNBC HOSPITAL, DELHI, INDIA
}

\author{
A. Manoharan ${ }^{1}$, V. Manchanda ${ }^{2}$, M. Deloria-Knoll ${ }^{3}$, G.D. Kahn³ ${ }^{3}$ K.L. O’Brien ${ }^{3}$
}

${ }^{1}$ Dept. of Medicine Unit I \& Infectious Diseases, Christian Medical College \& Hospital, Vellore, India; ${ }^{2}$ Dept. of Microbiology, Chacha Nehru Bal Chikitsalya, Delhi, India; ${ }^{3}$ International Vaccine Access Center, The Johns Hopkins Bloomberg School of Public Health, Baltimore, USA

Background: Pneumococcal disease data among Indian children is limited. We characterized children $<5$ years hospitalized with suspected IBD at CNBC Hospital, Delhi.

Methods: Children $<5$ years with suspected pneumococcal disease were enrolled prospectively September 2011- February 2013. 'Suspected pneumonia' was defined as cough, tachypnea, retractions, respiratory distress, breathlessness or chest-x-ray (CXR) taken. 'Suspected meningitis' was defined as fever plus physician-diagnosed meningeal signs or altered consciousness or cerebrospinal fluid (CSF) collected. Blood, CSF and pleural fluid specimens were cultured.

Results: Of 872 children enrolled, $68 \%$ and $85 \%$ were age $<1$ and $<2$ years; $99.8 \%$ had a CXR taken (abnormal=79.4\%), $17.8 \%$ had retractions; blood and pleural fluid was collected from $865(99.3 \%)$ and $24(2.8 \%)$, respectively. Of 255 (29\%) with suspected meningitis, blood, CSF and pleural fluid was collected from 249 (97.7\%), $227(89.0 \%)$ and $3(1.2 \%)$, respectively. Of $29(3.3 \%)$ pneumococci isolated from blood $(n=27)$ and/or CSF $(n=3)$, $37.9 \%$ and $51.7 \%$ were age $<1$ and $<2$ years, 9 (31.0\%) had suspected meningitis, $25(86.2 \%)$ had abnormal CXR, and 3 had WHO-defined alveolar consolidation (CXR-AC). Of 14 (1.6\%) Haemophilus influenzae isolated, 10 had suspected meningitis, 7 had abnormal chest-x-ray, and 1 had CXR-AC. Isolates were from blood $(n=9), \operatorname{CSF}(n=$ $5)$, or pleural fluid $(n=1)$. Staphylococcus aureus $(n=3)$, Escherichia coli $(n=2)$ and Salmonella typhi $(n=1)$ were also isolated. Of 45 deaths (5.2\%), 6 had a bacterium isolated: $S$. pneumoniae $=2, H$. influenzae $=2, S$. aureus $=1$ and Staphylococcus coagulase negative $=1$.

Conclusion: Pneumococcus was the most common pathogen isolated from children hospitalized with suspected IBD in this Delhi hospital. Isolation rates were similar to those observed elsewhere.

Conflict of interest

ISPPD-0405

Pneumococcal Pneumonia---Risky Business

\section{HAS THE IMPACT OF PNEUMOCOCCAL CONJUGATE VACCINATION ON PNEUMONIA HOSPITALISATION IN AUSTRALIA BEEN SUSTAINED?}

A. Jardine ${ }^{1}$, R. Menzies ${ }^{1}$, P. Mclntyre ${ }^{1}$

${ }^{1}$ Surveillance, National Centre for Immunisation Research and Surveillance, Westmead, Australia

Background and Aims: A 7vPCV program commenced in 2005 in Australia, with doses at 2, 4, and 6 months of age, and was associated with a significant reduction in hospitalisations for pneumonia in the first 2.5 years of the program. We updated our previous study to investigate the impact up to June 2011.

Methods: All hospital discharges in Australia with the primary diagnosis coded as pneumonia were included, between July 1998 and June 2011. Negative binomial regression modelling was used to quantify 7vPCV impact, as a ratio of post-vaccination and pre-vaccination hospitalisation rates, after adjusting for seasonal and pre-vaccine background trends.

Results: Significant decreases up to 6.5 years post-vaccination were seen in hospitalisations coded as pneumococcal and those with unspecified cause, while an increase was seen in those with other specified causes. For all-cause pneumonia, significant decreases in the $<2$ and 2-4 year age groups in the first 2.5 years post-vaccination were similar after the addition of another 4 years of data, but smaller decreases in older age groups were no longer significant (Table 1).

Table 1: Ratios of post-vaccination to pre-vaccination pneumonia hospitalisation rates, Australia, July 1998 to June 2011, adjusted for seasonal and pre-vaccine trends.

\begin{tabular}{|l|l|l|l|l|l|l|}
\hline Years post-vaccination & $<2$ years & $2-4$ years & $5-17$ years & $18-39$ years & $40-64$ years & $>65$ years \\
\hline $0-2.5$ & $0.62^{*}$ & $0.72^{*}$ & $0.89^{*}$ & $0.92^{*}$ & $0.96^{*}$ & 0.97 \\
\hline $0-6.5$ & $0.68^{*}$ & $0.80^{*}$ & 0.94 & 0.98 & 1.05 & 1.03 \\
\hline
\end{tabular}

*Statistically significantly below 1.0 , at $95 \%$ level

Conclusion: Decreases in all-cause pneumonia hospitalisations associated with the introduction of a 3+0 7vPCV schedule have been sustained up to 6.5 years post-vaccination for children $<5$ years of age, but not in older age groups.

No conflict of interest 
ISPPD-0455

Pneumococcal Pneumonia---Risky Business

\title{
CARRIAGE PREVALENCE, SEROTYPE DISTRIBUTION AND ANTIBIOGRAMS OF STREPTOCOCCUS PNEUMONIAE ISOLATES FROM THE PERCH STUDY, THE GAMBIA SITE
}

\author{
J. McLellan ${ }^{1}$, B. Ebruke ${ }^{1}$, R. Saluadeen ${ }^{1}$, E. Machuka ${ }^{1}$, S. Jarju ${ }^{1}$, M. Antonio ${ }^{1}$, S. Howie ${ }^{1}$, N. PERCH Study Group ${ }^{2}$ \\ ${ }^{1}$ Vaccinology/Child Survival, Medical Research Council Unit The Gambia, Fajara, Gambia; ${ }^{2}$ Johns Hopkins Bloomberg School of Public Health, \\ Baltimore, USA
}

Background and Aims: Streptococcus pneumoniae carriage among those with and without pneumonia provides insights into the evolving epidemiology of pneumococcal disease. We present data from the Gambian site of the Pneumonia Etiology Research for Child Health (PERCH) project.

Methods: Cases are children under 5 years with WHO-defined severe and very severe pneumonia and healthy controls from the community were frequency-matched for age and season. Nasopharyngeal-oropharyngeal (NP/ OP) swabs were collected from all participants and induced sputum (IS) was collected from cases. Culture and quantitative PCR (qPCR) were performed on all specimens, isolates were serotyped and antibiotic susceptibility testing performed on IS isolates.

Results: The carriage rate for $S$. pneumoniae was similar between cases and controls when NP/OP specimens were examined by culture or qPCR (85-90\%), which was comparable to previous local data. The prevalence of $S$. pneumoniae by culture was lower in IS $(66 \%, \mathrm{p}<.0001)$, than in NP/OP specimens but comparable when examined by qPCR (87\%). PCV13 vaccine coverage was $28 \%$ in NP/OP specimens, compared to $38 \%(p<0.001)$ prior to PCV13 introduction. PCV13 coverage was $31 \%$ for IS pneumococci. 19A, 6A/B 15B, 16F, 11A, and 35B were the predominant carriage serotypes. $23 \%$ of IS isolates showed complete or intermediate resistance to penicillin, the first-line drug for severe pneumonia in our setting. $16 \%$ of these isolates were additionally resistant to the secondline drug, chloramphenicol.

Conclusion: PCV13 appears to be influencing the serotype distribution of S. pneumoniae carriage. Resistance of IS pneumococci to standard antibiotics was seen in an appreciable minority.

No conflict of interest

ISPPD-0339

Pneumococcal Pneumonia---Risky Business

\section{SEROTYPE DISTRIBUTION OF PNEUMOCOCCAL COMMUNITY ACQUIRED PNEUMONIA IN GREECE: AN INTERIM ANALYSIS}

M. Gaga ${ }^{1}$, K. Gourgoulianis' ${ }^{2}$, D. Tsousis ${ }^{3}$, D. Menegas ${ }^{3}$, D. Antoniou ${ }^{4}$, V. Ioannidis ${ }^{5}$, A. Rapti ${ }^{6}$, A. Chaniotou ${ }^{7}$, U. Anagnostopoulou ${ }^{8}$, V. Filaditaki ${ }^{9}$, H. Moraitou ${ }^{10}$, S. Bousmoukilia ${ }^{11}$, G. Tatsis ${ }^{12}$, E. Petinaki ${ }^{13}$, G. Kouppari ${ }^{14}$, N. Georgatou ${ }^{15}$, K. Kanellakopoulou ${ }^{16}$, J. Trammel ${ }^{17}$, V. Tsekouras ${ }^{3}$, H. Giamarellou ${ }^{18}$

${ }^{1}$ Seventh Department of Pulmonary Medicine University of Athens, Chest Diseases Hospital “Sotiria”, Athens, Greece; ${ }^{2}$ Respiratory Clinic University Hospital of Larissa, University of Thessaly, Larissa, Greece; ${ }^{3}$ Medical Department, Pfizer Hellas, Athens, Greece; ${ }^{4}$ First Department of Pulmonary Medicine, Chest Diseases Hospital "Sotiria", Athens, Greece; ${ }^{5}$ First Pulmonology Clinic, General Hospital of Kavala, Kavala, Greece; ${ }^{6}$ Second Department of Pulmonary Medicine, Chest Diseases Hospital "Sotiria", Athens, Greece; 7 Pulmonary Department, "Amalia Fleming" General Hospital, Athens, Greece; ${ }^{8}$ Eighth Department of Pulmonary Medicine, Chest Diseases Hospital "Sotiria", Athens, Greece; ${ }^{9}$ Second Pulmonary Department, "Sismanoglio" General Hospital, Athens, Greece; ${ }^{10}$ Microbiology Laboratory, Chest Diseases Hospital "Sotiria", Athens, Greece ${ }^{11}$ Pulmonary Medicine Department, General Hospital of Kavala, Kavala, Greece; ${ }^{12}$ Pulmonary Medicine Department, Evaggelismos General Hospital, Athens, Greece; ${ }^{13}$ Department of Microbiology, University of Thessaly, Larissa, Greece; ${ }^{14}$ Microbiology Laboratory, "Amalia Fleming" General Hospital, Athens, Greece; ${ }^{15}$ Fifth Department of Pulmonary Medicine, Chest Diseases Hospital "Sotiria”, Athens, Greece; ${ }^{16}$ Fourth Department of Internal Medicine University of Athens, University General Hospital Attikon, Athens, Greece; ${ }^{17}$ inVentiv Health, Clinical, Princeton, USA ${ }^{18}$ Sixth Department of Internal Medicine, Hygeia General Hospital, Athens, Greece

Background: Streptococcus pneumoniae remains one of the major bacterial causes of community acquired pneumonia (CAP). The aim was to describe the serotype distribution in adults with chest X-ray confirmed pneumococcal CAP (pCAP) in Greece.

Methods: Interim analysis data from a prospective observational study conducted between January-May 2010 in adults $>18$ y old with clinicaly and radiographicaly confirmed CAP examined in the emergency departments of 15 secondary and tertiary hospitals are presented. Confirmation of $S$. pneumoniae was made by detection of specific antigen in the urine (BinaxNOW ${ }^{\circledR}$ Test) while further investigation for the serotypes included in the 13-valent pneumococcal conjugate vaccine (PCV13) with the urine antigen detection (UAD) assay was performed on the Luminex platform in Pfizer's central lab (Pearl River NY, USA).

Results: In total, 200 cases of X-ray confirmed CAP (mean age $51.4 \pm 20.5$ years) were recorded. Examination of the urine specimens revealed $12(6.0 \%)$ cases with a positive BinaxNOW ${ }^{\circledR}$ Test and $21(10.5 \%)$ UAD positive cases of which only $9(42.9 \%)$ were also positive with the BinaxNOW ${ }^{\circledR}$ Test. In addition 3 BinaxNOW ${ }^{\circledR}$ positive cases were negative with the UAD test. Among the 24 pCAP cases detected by non-cultural methods, $21(87.5 \%)$ were caused by serotypes included in PCV13. The most prevalent serotype was $3(8 / 21,38.1 \%)$ followed by $7 F(5 / 21,23.8 \%), 19 \mathrm{~A}$ $(4 / 21,19.0 \%)$ and $23 \mathrm{~F}(1 / 21,4.8 \%)$.

Conclusion: The majority of pCAP cases in this study seem to be attributed to PCV13 serotypes. Non-cultural methods and specifically UAD testing may provide a better means to detect PCAP. 
ISPPD-0141

Pneumococcal Pneumonia---Risky Business

\title{
IMPORTANCE OF PNEUMOCOCCAL ETIOLOGY IN ADULT SEVERE LOWER RESPIRATORY TRACT INFECTIONS IN PRIMARY CARE IN BELGIUM
}

\author{
J. Flamaing ${ }^{1}$, S. Heijmans ${ }^{2}$, Y. Van Laethem ${ }^{3}$, A. Mignon ${ }^{4}$ \\ ${ }^{1}$ Geriatrics, UZ Leuven, Leuven, Belgium; ${ }^{2}$ General Practice, Research link, Linkebeek, Belgium; ${ }^{3}$ Infectious Diseases, CHU St Pierre, Brussels, \\ Belgium; ${ }^{4}$ Medical, Pfizer, Brussels, Belgium
}

Background and Aims: Pneumococcal disease is largely underrecognized in general practice. We aimed to evaluate the percentage of Streptococcus pneumoniae in severe lower respiratory tract infection (SLRTI) in primary care using commercial urinary antigen detection test and to evaluate the profile of patients, clinical signs, healthcare use and loss of functionality of pneumococcal SLRTI in comparison to non-pneumococcal SLRTI.

Methods: Multi-center prospective epidemiological study on SLRTI in primary care. Patients were included based on clinical signs of SLRTI. Pneumococcal infection was identified by urinary antigen detection (BinaxNow). Investigators were blinded to the results. BinaxNow-positive cases and BinaxNow-negative controles were followed-up after 4 weeks and compared.

Results: 549 patients were enrolled by 38 general practitioners (GPs) between 27 February 2011 and 28 March 2013. BinaxNOW test was positive in 26 patients (4.7\%). 25 of the positive cases and 60 negative controles were compared. In the positive patients there were more women $(p=0.016)$, less exposure to young children $(p=0.009)$, more patients without revenue or with a replacement revenue $(p=0.031)$, less seasonal influenza vaccination $(p=$ $0.037)$ and more COPD $(p=0.002)$. More clinical signs were present and self-rated health was lower. The number of GP visits $(p=0.001)$, number of working days loss $(p<0.0001)$ and number of assist days were higher $(p=0.014)$. Conclusion: Currently available urinary antigen detection methods identify pneumococcal infections in a limited number of LRTI patients in primary care but these patients have more symptoms and impairment and require more medical care.

The study was sponsored by Pfizer.

Conflict of interest

ISPPD-0479

Pneumococcal Pneumonia---Risky Business

\section{COMMUNITY ACQUIRED PNEUMOCOCCAL PNEUMONIA LEADS TO CHALLENGING MULTIDRUG RESISTANT GRAM NEGATIVE INFECTION}

\author{
D.N. Mukherjee ${ }^{1}$ \\ ${ }^{1}$ Department of Microbiology, Woodland Hospital, Kolkata, India
}

Community-acquired pneumonia (CAP) is caused by several pathogens and Streptococcus pneumoniae is one of them. It causes CAP in older adults and can lead to serious life threatening secondary multidrug resistant Gram negative infection, even in older adults. A 66 y old male patient, known diabetic, hypertensive, chronic obstructive pulmonary disease, presented with fever, confusion, pleuritic chest pain, respiratory distress with cough and bloodtinged expectoration. On examination: tachypnoea, right basal creps+, CURB 65 score 4. Admitted for investigation and treatment. X-ray: right lower pneumonia, total leukocyte count (TLC) 16500 (Neutrophil 92\%), C-reactive protein (CRP) 116. Considering a case of CAP, emperic antibiotic started inj Pip-tazo4.5 gm IV three times daily (TDS) +Azithromycin 500mg daily, after collecting sputum and blood culture. Patient needed mechanical ventilation on day 2 for worsening dyspnea and hypoxia. Sputum Gram stain: plenty pus cells, occasional epithelial cells and fair number of gram positive encapsulated diplococci likely pneumococci. Culture-mixed growth. Pneumococcal pneumonia likely. Treatment continued. Re-onset of fever on day 6 with tachypnea and hypoxia. Reintubated, increasing TLC (19000), endotracheal (ET) secretion+, repeat X-ray found new opacity in left lower lung field. Procalcitonin 5, CRP 195. Considering case of ventilator-associated pneumonia (VAP), antibiotic escalation done to Meropenem at 1gm Intra-venous (IV) TDS, after collecting ET suction and blood for culture. Culture grew Acinetobacter baumannii, sensitive only to colistin/poly B. Colistin added with the existing regime 9 million units (MU) loading, followed by $2 \mathrm{MU}$ TDS according to creatinine clearance, cosidering synergistic effect. Patient became afebrile within the next 48 hours, followed by successful weaning off ventilator. Antibiotic therapy continued for a total of 7 days. Patient was successfully discharged on day 16 with complete microbiological clearance. Being a life threatening organism itself, this pathogen may also help other resistant Gram negative pathogens to be established in a previously infected host. So prevention of primary infection can avoid serious secondary infection in older adult.

No conflict of interest 
ISPPD-0010

Pneumococcal Pneumonia---Risky Business

\title{
STREPTOCOCCUS PNEUMONIAE CIPL INHIBITS ADHERENCE TO A549 HUMAN LUNG CELLS THROUGH RAP1- MEDIATED ACTIN REMODELING
}

\author{
C.T. Nguyen ${ }^{1}$, T.N. Le ${ }^{1}$, T.D.H. Tran' ${ }^{1}$, E.H. Kim¹, K.T. Chung ${ }^{2}$, S. Pyo ${ }^{1}$, D.K. Rhee ${ }^{1}$ \\ ${ }^{1}$ School of Pharmacy, Sungkyunkwan University, Suwon, Korea; ${ }^{2}$ Department of Clinical Laboratory Science, Dong-Eui University, Busan, Korea
}

Caseinolytic Protease $L(C / p L)$ is a member of the HSP100/Clp chaperone family found mainly in Gram-positive bacteria. During infection, ClpL is highly expressed to refold stress-induced denatured proteins at least some of which are important for adherence. However, the mechanism by which $\mathrm{ClpL}$ modulates pneumococcal virulence is poorly understood. Here, we investigate how ClpL inhibits the adherence of Streptococcus pneumoniae (D39) to A549 human lung cells. Using microarray and Western blot analyses, ClpL was found to induce and activate small GTPases (Rap1, Rac1, Cdc42, and Rho) and decrease the ratio of cofilin:p-cofilin, which regulates actin dynamics. Thus, actin re-organization and cytoskeleton re-arrangement were increased in clpL mutant (HYK304) infected cells compared to D39 wild type infected cells. Filament formation was also higher in HYK304 infected cells than D39 wild type infected cells. Rap1- and Rac1-deficient cells showed impaired filament formation and decreased $S$. pneumoniae adherence to A549 cells. These results demonstrate a novel role of ClpL in pneumococcal resistance to adherence to host cells during infection, and provide insight into further understanding the interactions between hosts and pathogens.

No conflict of interest

ISPPD-0204

Pneumococcal Pneumonia---Risky Business

\section{INTER- AND INTRA-OBSERVER VARIATION ANALYSIS OF CHEST RADIOGRAPHS FOR RADIOLOGICALLY-CONFIRMED PNEUMONIA}

M.S. Ominde ${ }^{1}$, J.A. Sande ${ }^{2}$, J. Ignas ${ }^{3}$, J. Ojal ${ }^{1}$, K. Maitland ${ }^{1}$, K. Park ${ }^{4}$, R. Benamore ${ }^{5}$, F. Gleeson ${ }^{5}$, J.A.G. Scott ${ }^{1}$

${ }^{1}$ Epidemiology and Demography, KEMRI-Wellcome Trust Research Programme, Kilifi, Kenya; ${ }^{2}$ Imaging and Diagnostic Radiology, Aga Khan University Nairobi, Nairobi, Kenya; ${ }^{3}$ Clinical, KEMRI-Wellcome Trust Research Programme, Kilifi, Kenya; ${ }^{4}$ John Radcliffe Hospital, Oxford University Hospitals NHS Trust, Oxford, United Kingdom; ${ }^{5}$ Churchill Hospital, Oxford University Hospitals NHS Trust, Oxford, United Kingdom

Background and Aims: Radiologically-confirmed pneumonia (RCP) has been used as an important end-point in pneumococcal vaccine trials. Reporting of radiographs is subject to individual variation. Agreement statistics are used to validate such studies. In this study, we aimed to compare three measures, Cohen's kappa, AC1 and percent agreement.

Methods: A team of clinicians and radiologists attended a three-day training on CXR interpretation for RCP using a WHO standard. A set of 4 questions was used to interpret each radiograph. 2100 chest radiographs from children with WHO-defined severe and very severe pneumonia were read. Initially, two readers reported all the radiographs independently. All discordant results and $10 \%$ of concordant readings progressed to the second level of arbitration. In the initial readings a repeat subset of $5 \%$ was introduced randomly to assess intra-observer variation. Sensitivity and specificity were tested on the first level readers against the arbiters' panel readings, which were considered to be the 'gold standard'.

Results: We analyzed 2096 radiographs since 4 had incomplete fields. The percent agreement, kappa and AC1 on RCP were $89 \%, 0.63$ and 0.84 and ranged between $79-100 \%, 0.17-0.89$ and $0.84-1.00$ on all reading criteria, respectively. Both level one readers had kappa $>0.7$ for intra-observer variability. Sensitivity ranged from $65-87 \%$ and specificity from $86-99 \%$.

Conclusion: After training on the WHO interpretive standard, the agreement between two readers is relatively high, and repeatability is moderately good. However, upto $1 / 3$ of 'true' cases of pneumonia may be missed and up to $14 \%$ of normal radiographs misclassified as pneumonia.

No conflict of interest 
ISPPD-0006

Pneumococcal Pneumonia---Risky Business

\title{
ENVIRONMENTAL TOBACCO SMOKE AS A RISK FACTOR TO INCREASING RESPIRATORY CHILDHOOD INFECTION AND PNEUMONIA IN SOUTH WEST NIGERIA
}

\author{
S. Omiyefa ${ }^{1}$ \\ ${ }^{1}$ Programs, Youth Action on Tobacco Control and Health, Ibadan, Nigeria
}

Background and Aims: There is consistent evidence that children exposed to environmental tobacco smoke (ETS) have higher incidence of asthma, ear- and throat disease, worsening of asthma symptoms and lung symptoms as cough, wheezing and pneumonia, The objective of this study is to consider exposure to ETS as a risk factor to increasing respiratory childhood infection and pneumonia in the South West region Nigeria.

Materials and Methods: A descriptive cross sectional study was carried out in 5 states (Ogun, Lagos, Akure, Oyo and Ekiti). A multistage cluster random sampling was employed to select 450 respondents in each state. Data was collected using structured questionnaires by trained interviewers.

Results: About 2113 records were available for analysis. There were 1298 (60.7\%) males and 815 (38.1\%) females. A Majority, 807 (38.0\%) live with both parents, 213 (10.0\%) live with mother alone while 265 (12.5\%) live with relatives. Analysis was done using SPSS version 15 and the prevalence of exposure to ETS was determined. The prevalence of children exposed to ETS in the southwest region Nigeria was $73.2 \%$, the study further revealed that $28.5 \%$ of children in this region with respiratory childhood infection are exposed to environmental tobacco smoke and $18.4 \%$ pneumonia cases are attributed to ETS. However, $(122,14.7 \%)$ parents/relatives don't see a problem with using cigarettes or any tobacco products. It is clearly stated that about $46.9 \%$ cases of respiratory childhood infection and pneumonia combined are attributed to ETS in the South West region Nigeria.

Conclusion: Since ETS has this much negative effects on children in the South West region Nigeria. Efforts should be tailored towards protecting children from ETS to reduce the rate of children exposed to ETS, thereby curbing or reducing respiratory childhood infection and pneumonia in Nigeria.

No conflict of interest

ISPPD-0168

Pneumococcal Pneumonia---Risky Business

\section{PRELIMINARY RESULTS OF MADAGASCAR SITE OF THE PILOT PNEUMONIA MULTICENTRIC STUDY OF THE GABRIEL NETWORK, FONDATION MÉRIEUX}

M.N. Maeder ${ }^{1}$, H. Rabezanahary ${ }^{1}$, N. Zafindraibe ${ }^{1}$, A. Robinson², V. Picot ${ }^{3}$, M. Messaoudi ${ }^{3}$, J.N. Telles ${ }^{3}$, F. Komurian-Pradel ${ }^{3}$, B. Contamin ${ }^{1}$, G. Paranhos-Baccalà ${ }^{3}$

${ }^{1}$ Centre d'Infectiologie Charles Mérieux Université d'Antananarivo, Fondation Mérieux, Antananarivo, Madagascar; ${ }^{2} \mathrm{CHU}$ d'Antananarivo Mère et Enfant Tsaralalana, Fondation Mérieux, Antananarivo, Madagascar; ${ }^{3}$ Emerging Pathogens Laboratory, Fondation Mérieux, Lyon, France

Background and Aims: First site of the Pilot Multi-centric Pneumonia Study in the GABRIEL network, the patient enrollment was launched in December 2010. The final purpose is to provide information on the pneumonia etiology (viruses and bacterial) to improve case management of the pneumonia ill child in Madagascar.

Methods: A hospital-based case-control study has been carried-out in one center located in Antananarivo. Less than 3 years are needed to enroll 123 cases and 103 controls under 5 years of age. Respiratory specimens and whole blood were collected from all pneumonia cases and nasal swabs for controls. The viruses and bacteria were identified using real-time multiplex PCR assays (Respiratory 21+, Fast-Track diagnostics). Streptoccus pneumoniae was serotyped in positive samples.

Results: The main bacterial agent found in cases and controls respiratory samples were S. pneumoniae (83\% and $72 \%$, respectively). Staphylococcus aureus, Haemophilus influenzae b, Mycoplasma pneumoniae and Chlamydia pneumoniae were less represented ( $16 \%$ to $0 \%)$. For the viral population, respiratory syncytial virus (RSV) was the principal agent found ( $15 \%$ in cases and only at $2 \%$ in controls). Human metapneumovirus (hMPV), parainfluenza-3 (PIV-3) and Influenza-A/B were found mainly in cases. Rhinoviruses (28\%), Bocaviruses (10\%) and Enteroviruses (9\%) were found equally in controls and cases. The main S. pneumoniae serotypes represented were 6A/B, 5, 19F and in less proportion 14, and 19A.

Conclusion: This study allows a description of the etiologies of pneumonia among hospitalized children in Madagascar. The results should permit a better implementation of targeted curative or preventive measures.

No conflict of interest 
ISPPD-0135

Pneumococcal Pneumonia---Risky Business

\title{
THE SEROTYPE DISTRIBUTION OF ADULT NON-INVASIVE PNEUMOCOCCAL PNEUMONIA CHANGED IN THE ERA OF CHILDHOOD VACCINATION
}

\author{
A.N. das Neves Horácio ${ }^{1}$, M. Ramirez ${ }^{1}$, J. Melo-Cristino ${ }^{1}$ \\ ${ }^{1}$ Instituto de Microbiologia, Faculdade de Medicina da Universidade de Lisboa, Lisbon, Portugal
}

Background and Aims: Non-invasive pneumococcal pneumonia (NIPP) is a frequent cause of morbidity and mortality among adults. We aimed at determine the serotype distribution of the isolates causing NIPP in adults ( $\geq 18$ y) and the herd effect of childhood vaccination.

Methods: A total of 1300 NIPP isolates collected between 1999 and 2011 (100 isolates/year, chosen randomly) were serotyped and tested for antimicrobial resistance.

Results: Serotype diversity was high for all studied years (Simpson's index of diversity $>0.9$ ). Overall, the most common serotypes were $3(18 \%), 11 \mathrm{~A}(7 \%), 19 \mathrm{~F}(7 \%), 14(4 \%), 23 \mathrm{~F}(4 \%)$ and $9 \mathrm{~N}(4 \%)$. The serotypes that were consistently recovered in NIPP throughout the study years were serotypes 3, 11A and 19F. Between 1999 and 2011 there was a strong reduction in the serotypes included in PCV7 (from $23 \%$ to $11 \%$ ), PCV10 (from $28 \%$ to $14 \%$ ) and PCV13 (from 58\% to 44\%). Individually, serotypes 6B, 9V and 23F decreased while serotype 8 increased. When comparing the serotypes of NIPP and IPD immediately prior to the availability of PCV13 for adults (2009-2011) only serotypes 3 and 19A were amongst the most common in both infections. While PCV13 potential coverage in IPD was 59\%, in NIPP it was only 43\%. Between 1999 and 2011 erythromycin resistance of NIPP isolates increased from $8 \%$ to $18 \%$.

Conclusion: Similarly to what happened in adult IPD, there was an effect in adult NIPP of PCV7 childhood vaccination. The serotype distribution of contemporary NIPP and IPD isolates was different.

No conflict of interest

ISPPD-0528

Pneumococcal Pneumonia---Risky Business

\begin{abstract}
CHALLENGES AND OPPORTUNITIES IN SETTING UP A SURVEILLANCE NETWORK FOR DETECTING INVASIVE PNEUMOCOCCAL DISEASE IN INDIA: PIDOPS NETWORK-PRELIMINARY DATA
\end{abstract}

G. Vandana ${ }^{1}$, A. Feroze Ganaie ${ }^{1}$, K.L. Ravikumar ${ }^{1}$

${ }^{1}$ Microbiology, Kempegowda Institute of Medical Sciences, Bangalore, India

Background and Aims: The country epidemiology data of invasive Streptococcus pneumoniae is sparse and is limited by low sensitivity of the culture methods, the gold standard in diagnosing the invasive infection and inadequate serotype data.The 'Pan India Distribution Of Pneumomococcal Serotypes' (PIDOPS) study network is designed to identify the characteristics of invasive pneumococcal disease in children by combing the culture methods with molecular techniques and by serotyping the isolates amassed across the centers.

Methods: The hospital based prospective observational study is being conducted in 4 hospitals in India: KIMS Bangalore, DMC Ludhiana, UMC Jodhpur and ICH Chennai. Children under 5 years of age presenting with clinically suspected IPD or pneumonia before the initiation of empirical antibiotic therapy were enrolled. POI (point of interest) software is used to standardize and collect the e-CRF in real time.Laboratory facility for RT-PCR detection of S.pneumoniae, conventional, molecular serotyping and archiving is established at Central Research Laboratory, Bangalore.

Results: From 13 September 2013 to 15 October 2013, 65 children were enrolled in the study from four participating centers. 60 of them were presented with pneumonia and 5 with meningitis. There were 2 culture positives and 7 with real-time PCR (RT-PCR) out of 29 samples collected in KIMS center.

Conclusion: The preliminary data suggest that the approach of combining culture techniques with molecular methods will improve the identification of pneumococcal infections in children. This prospective study is expected to provide critical data on pneumococcal infection prevalence and its serotypes in a representive Indian population that will support public health policy development in India.

No conflict of interest 
ISPPD-0402

Pneumococcal Pneumonia---Risky Business

\title{
EFFECT OF INFANT IMMUNIZATION ON PNEUMONIA WITH ALVEOLAR CONSOLIDATION BY AGE: COMPARISON OF DOUBLE-BLIND RANDOMIZED CONTROLLED TRIALS
}

\author{
J. Ruiz Guiñazú1 ${ }^{1}$ M. Moreira ${ }^{1}$, M.W. Tregnaghi ${ }^{2}$, S.A. Madhi ${ }^{3}$, W.P. Hausdorff ${ }^{1}$, D. Borys ${ }^{1}$
}

${ }^{1}$ Vaccine Discovery and Development, GlaxoSmithKline Vaccines, Wavre, Belgium; ${ }^{2}$ Centro de Desarrollo de Proyectos Avanzados en Pediatría, (CEDEPAP), Córdoba, Argentina; ${ }^{3}$ Department of Science/National Research Foundation: Vaccine Preventable Diseases and Medical Research Council: Respiratory and Meningeal Pathogens Research Unit, University of the Witwatersrand, Johannesburg, South Africa

Background and Aims: Several double-blind randomized controlled trials (DBRCTs) with pneumococcal conjugate vaccines (PCVs) demonstrated similar point estimates of vaccine efficacy (VE; range: 23-37\%) against pneumonia with alveolar consolidation following infant immunization. We examined the duration of VE by age in each DBRCT. Methods: We reviewed DBRCTs to compare per-protocol efficacy of different PCVs following infant immunization against first episodes of community-acquired pneumonia with alveolar consolidation (AC-CAP, using the standardized WHO definition where available) by age (Table).

Results: VE appeared to be maintained ( $>20 \%$ ) through the third year of life with 9vCRM in the Gambia and Soweto, and with PHiD-CV in Latin America (Table). In contrast, waning of VE against AC-CAP was observed after the first year of life in the Northern California Kaiser Permanente trial with 7vCRM and in the Bohol trial with 11vDT.

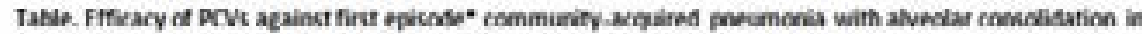
children by afe group reported in different DBRCTis fper protocel analyes)

\begin{tabular}{|c|c|c|c|c|c|c|}
\hline \multirow{2}{*}{ stude } & \multirow{2}{*}{$\begin{array}{l}\text { Vactire } \\
\text { Siledule }\end{array}$} & \multirow{2}{*}{$\begin{array}{l}\text { Ace growa } \\
\text { (monita) }\end{array}$} & \multirow[b]{2}{*}{$n$} & \multicolumn{2}{|c|}{ Number of epirodet } & \multirow{2}{*}{$\begin{array}{l}\text { Veccile etriceory } \\
195 \% \mathrm{cos}\end{array}$} \\
\hline & & & & rev & Consul & \\
\hline \multirow{5}{*}{ 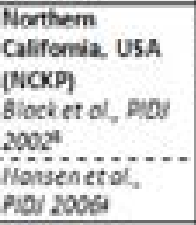 } & \multirow{5}{*}{$\begin{array}{l}\text { NCQM } \\
2,4,6 \\
12-15 \text { me }\end{array}$} & $6.5-11$ & \multirow{5}{*}{33,766} & ma & NA & $32.2 \mathrm{~N}(9.3 ; 525)^{\mathrm{T}}$ \\
\hline & & $12=23$ & & NA & NA & 20.00sion \\
\hline & & $24-42$ & & ma & NA & $9.1 \%(0.09,16.8)^{3}$ \\
\hline & & Qwarall. & & Ma. & NA & $20.5 \mathrm{~K} ; 4,4 ; 24.0 \%$ \\
\hline & & Overill & & $\mathrm{Ma}$ & NA & $30.3 \times[10.7 ; 45.7)$ \\
\hline \multirow{2}{*}{$\begin{array}{l}\text { Doha. The } \\
\text { Pmitipeines } \\
\text { turevo ef er, Mrar } \\
\text { joov }\end{array}$} & \multirow{2}{*}{$\begin{array}{l}\text { IlvDT } \\
6.10,14 \\
\text { nos: }\end{array}$} & $4-11$ & \multirow{2}{*}{12,031} & 47 & $\pi$ & $34.08(4.8 ;(5.3)$ \\
\hline & & $12-23$ & & 50 & 51 & $2.7 \%(43.5 ; 34.0)$ \\
\hline \multirow{5}{*}{ 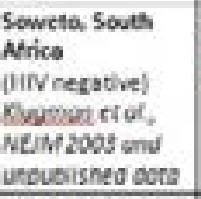 } & \multirow{5}{*}{$\begin{array}{l}\text { 9rCRM } \\
6,10,14 \\
\text { eds. }\end{array}$} & $4-11$ & \multirow{5}{*}{94.700} & 97 & 75 & $27.9 \times(-2): 45.7)$ \\
\hline & & $12-21$ & & 48 & 6 & $23.8 \times(-1,1 ; 47.7)$ \\
\hline & & $24-55$ & & 20 & 27 & $26.0 \mathrm{~N}(32.0: 58.5)$ \\
\hline & & $36-47$ & & 12 & 13 & $7.7 \times(192.2,57.9)$ \\
\hline & & $45-72$ & & 8 & 7 & $-14.2 \%(2215.0 ; 55.6]$ \\
\hline \multirow{3}{*}{$\begin{array}{l}\text { Tha Gambia } \\
\text { Catts et of, } \\
\text { Loncet } 2005\end{array}$} & \multirow{3}{*}{$\begin{array}{l}\text { 9.CRM } \\
6,10,14 \\
\text { weld }\end{array}$} & $4-11$ & \multirow{3}{*}{16.345} & 124 & 188 & $35 \%(12,48)$ \\
\hline & & $12-23$ & & 181 & 285 & $38 \%(25: 49)$ \\
\hline & & $24-29$ & & 28 & 40 & $375(-10,59)$ \\
\hline \multirow{4}{*}{$\begin{array}{l}\text { Latin America } \\
\text { [Compas] }\end{array}$} & \multirow{4}{*}{$\begin{array}{l}\text { Prio CV } \\
2,4,6, \\
15-18 \text { me }\end{array}$} & 6.511 & \multirow{4}{*}{20,351} & 53 & 62 & $15.1 \%(22.5,42.2)$ \\
\hline & & $12-23$ & & os & 99 & $31.85: 17.2: 49.9)$ \\
\hline & & $20-15$ & & 48 & b) & $20.78(-15.9 ; 45.8)$ \\
\hline & & $36-40$ & & 12 & 10 & $-19.3 \%(-175,2,45.5)$ \\
\hline \multicolumn{7}{|c|}{ 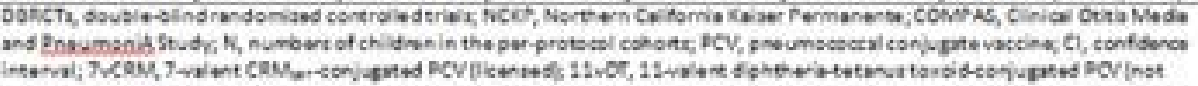 } \\
\hline \multicolumn{7}{|c|}{ 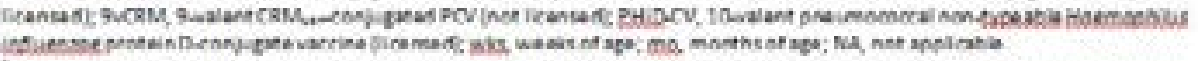 } \\
\hline \multicolumn{7}{|c|}{ 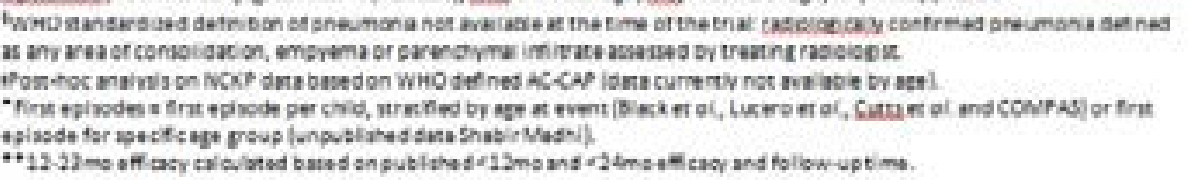 } \\
\hline
\end{tabular}

Conclusion: There is evidence that 9vCRM and PHiD-CV, but not 7vCRM or 11vDT, provided extended protection against AC-CAP through the third year of life. The ability of some PCVs to maintain efficacy through 3 years of age does not seem to correlate with immunogenicity or serotype composition, suggesting the trend for an agedependent decline for some vaccines might be due to other factors such as inter-site variations in pneumonia etiology, disease severity, or care-seeking patterns.

Funding: GlaxoSmithKline Biologicals SA

Conflict of interest 
ISPPD-0151

Pneumococcal Pneumonia---Risky Business

\title{
IMPACT OF INTRODUCTION OF PNEUMOCOCCAL CONJUGATE VACCINE (PCV) ON PNEUMONIA DISEASE BURDEN AND MORTALITY IN BANGLADESH
}

\author{
S. Saha ${ }^{1}$, S. Black ${ }^{2}$, F. Petraglia ${ }^{3}$
}

${ }^{1}$ Dhaka Shishu Hospital Department of Microbiology, Child Health Research Foundation, Dhaka, Bangladesh; ${ }^{2}$ Pediatrics, Center for Global Health, Ohio, USA; ${ }^{3}$ Obstetrics and Gynecology, University of Siena, Siena, Italy

Introduction: Pneumonia causes substantial child mortality and morbidity in absence of PCV in Bangladesh. The study aimed to estimate possible impact of PCV on pneumonia disease burden and overall mortality among children aged 2-59 months following introduction of PCV in Bangladesh.

Methods: The study estimated PCV preventable pneumonia morbidity and mortality based on pneumonia disease burden in Bangladesh and PCV effectiveness demonstrated in other countries. Methods of estimating PCV preventable clinical pneumonia cases, hospitalizations and deaths, and overall deaths are shown in Figure-1. Sensitivity analyses were performed using available data and different approaches to ascertain uncertainty range. Figure-1:

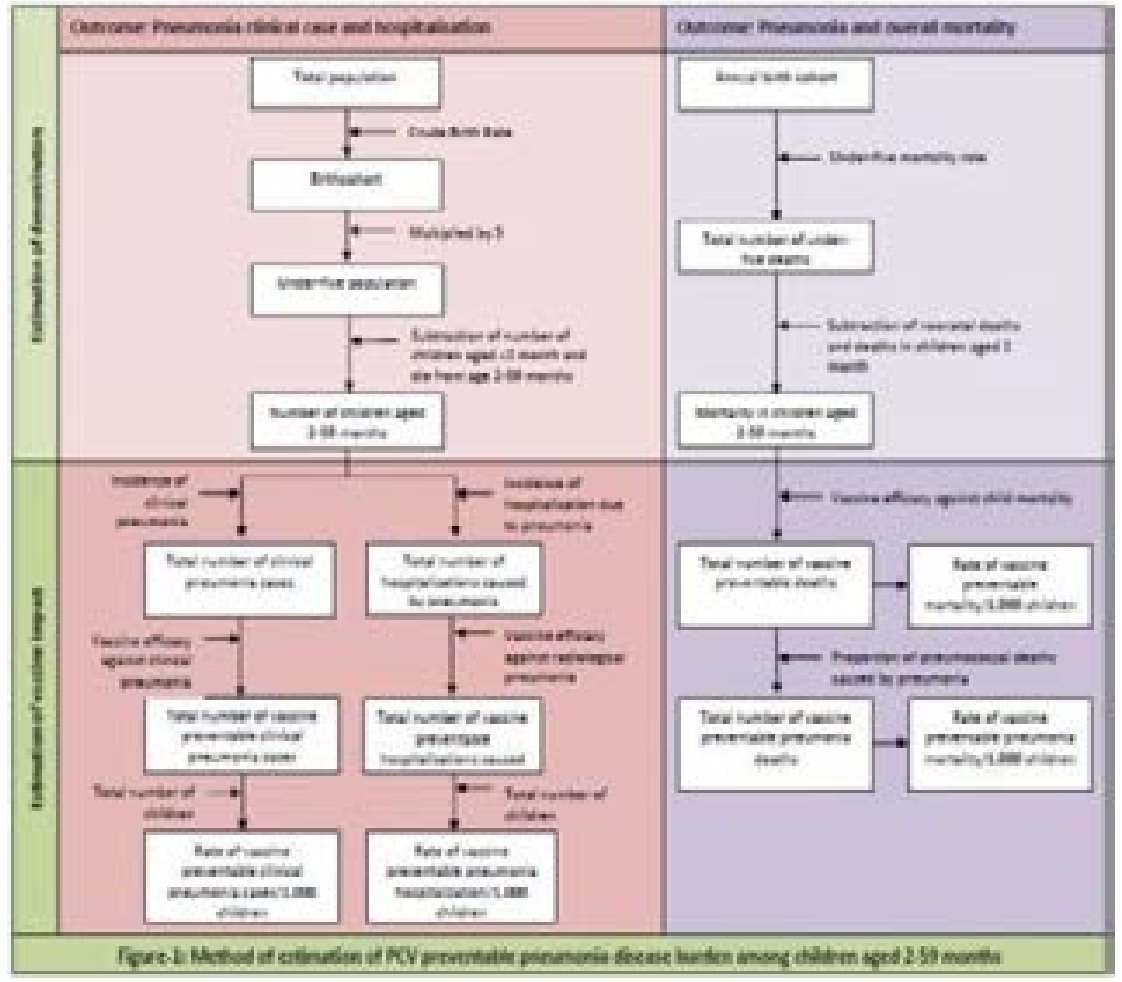

Results: PCV preventable pneumonia mortality and morbidity estimated in this study have been summarized in Table-1 and Figure-2.

Table 1: PCV preventable pneumonia disease burden and mortality/year

\begin{tabular}{|l|l|l|}
\hline Outcome & $\begin{array}{l}\text { Estimated number } \\
\text { (uncertainty range) }\end{array}$ & $\begin{array}{l}\text { Rate/1,000 children } \\
\text { (uncertainty range) }\end{array}$ \\
\hline $\begin{array}{l}\text { Preventable clinical pneumonia } \\
\text { cases }\end{array}$ & $547,558(444,323-547,558)$ & $35(29-35)$ \\
\hline $\begin{array}{l}\text { Preventable pneumonia } \\
\text { hospitalisations }\end{array}$ & $183,922(183,922-366,081)$ & $11.9(11.9-23.6)$ \\
\hline Preventable pneumonia deaths & $9,264(3,011-9,264)$ & $0.6(0.2-0.6)$ \\
\hline Preventable overall mortality & 10,293 & 0.7 \\
\hline
\end{tabular}


Figure 2:

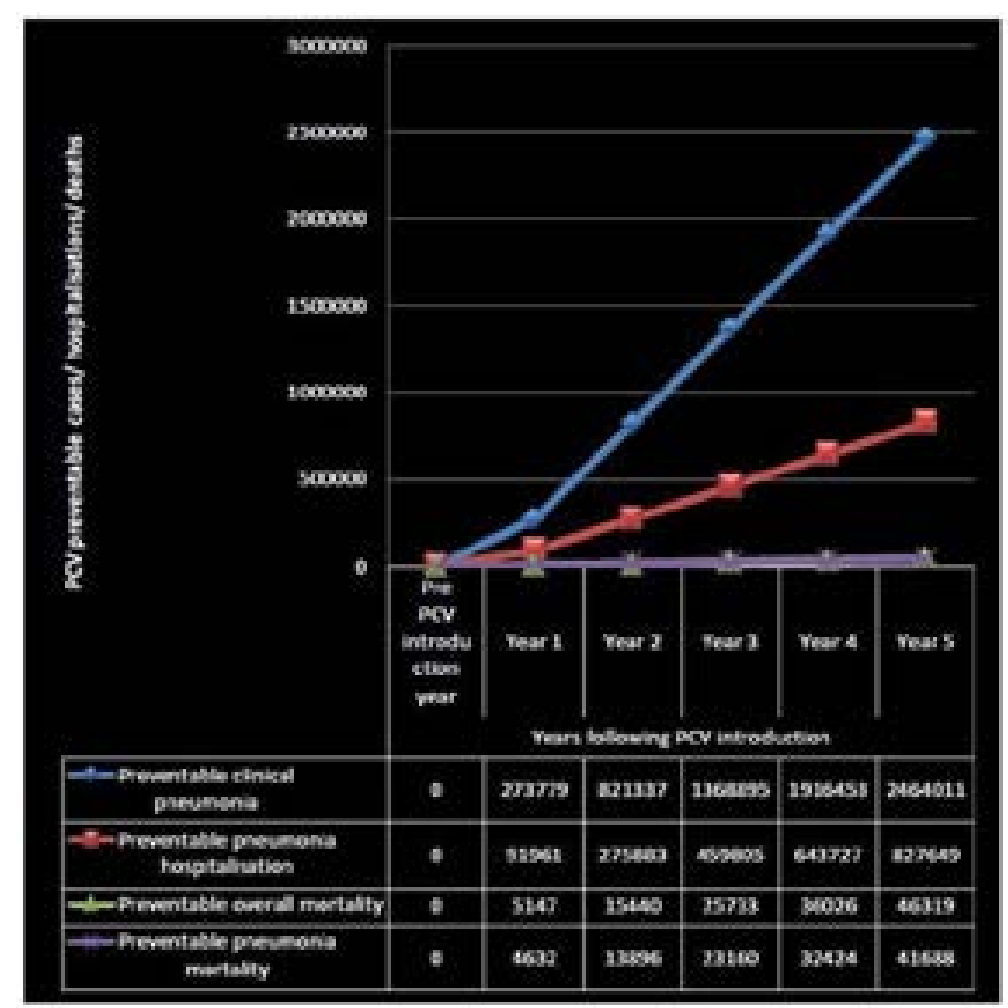

Ficure-2i Graph showine cumulative imcact of FCV wo pnecmonia disease burdes and martalty among children aged 2.59 months ahter PCV introduction

Conclusion: Introduction of PCV would have a great public health impact in Bangladesh. Studies are required to test the study results after introduction of PCV in the country.

Acknowledgment: University of Siena and Novartis Vaccine Academy, Italy; Samir K Saha.

No conflict of interest

\section{ISPPD-0242}

Pneumococcal Pneumonia---Risky Business

\section{SEROTYPE DISTRIBUTION OF INVASIVE STREPTOCOCCUS PNEUMONIAE IN CANADA, 2012}

R. Singh ${ }^{1}$, W. Demczuk ${ }^{1}$, A. Griffith ${ }^{1}$, I. Martin ${ }^{1}$, . Canadian Public Health Laboratory Network ${ }^{1}$

${ }^{1}$ National Microbiology Laboratory, Public Health Agency of Canada, Winnipeg, Canada

Background: The 13-valent pneumococcal conjugate vaccine (PCV13) was introduced in Canada during 2010. This study presents the distribution of serotypes of invasive Streptococcus pneumoniae in Canada during 2012.

Methods: A total of 1653 isolates of $S$. pneumoniae from sterile clinical sites (blood, $n=1513$; cerebral spinal fluid, $n$ $=61$; pleural fluid, $n=28$; other sterile sites, $n=51$ ) were serotyped in 2012. Isolates were aggregated by age into: $0-14$ years $(n=232)$; adults $15-64$ years $(n=802)$; seniors $\geq 65$ years $(n=603)$; unknown age $(n=16)$. Serotypes were determined by Quellung reaction using commercial antisera.

Results: PCV13 serotypes 7F and 19A are most prevalent overall together accounting for $25 \%$ of isolates in 2012 ; representing $30 \%(n=70)$ of isolates from children $<15$ years of age; $28 \%(n=227)$ of isolates from adults $15-64$ years of age; and $19 \%(n=112)$ from seniors $\geq 65$ years of age. Non-PCV13 serotype $22 \mathrm{~F}$ is the next most prevalent serotype overall with of $11 \%(n=187)$ of total isolates; $9 \%(n=21)$ of children aged $<15$ years; $9 \%(n=75)$ of adults aged 15-64; and $15 \%(n=88)$ of seniors aged $\geq 65$ years. Another PCV-13 serotype 3 was also prevalent in all age groups, consisting of $8 \%(n=136)$ of the isolates overall, $10 \%(n=23)$ of isolates from children $<15$ years of age, $8 \%$ $(n=61)$ of isolates from adults $15-64$ years of age, and $9 \%(n=52)$ of isolates from seniors $\geq 65$ years of age.

Conclusion: Continued surveillance of $S$. pneumoniae will be important to recognize replacement serotypes and assess the effectiveness of PCV13 in reducing invasive pneumococcal disease in Canada.

No conflict of interest 
ISPPD-0188

Pneumococcal Pneumonia---Risky Business

\title{
INCIDENCE OF VENTILATOR-ASSOCIATED PNEUMONIA AND ANTIBIOTIC SUSCEPTIBILITY OF CENTRAL CHINESE REGIONS: A RETROSPECTIVE STUDY
}

\author{
T. Sosorburam ${ }^{1}$, M. Chen Xie ${ }^{2}$, X. Wang ${ }^{3}$, X. Bai Su ${ }^{4}$ \\ ${ }^{1}$ Intensive Care, Union Hospital, Wuhan, China; ${ }^{2}$ Microbilogy, Tongji Medical College, Wuhan, China; ${ }^{3}$ Intensive Care, Chendu Medical college, \\ Chengdu, China; ${ }^{4}$ Intensive Care, Reming Hospital, Pekin, China
}

Background: Respiratory tract infections are the most common in intensive care units (ICU), with a high rate of mortality. The aim of the study was to identify the incidence of ventilator-associated pneumonia (VAP), antibiotic susceptibility and short-term outcome in ICU.

Method: The study was carried out over a period of 14 months in five ICU departments of Chinese hospitals. The patients who had a respiratory tract infection diagnosed in the first 5 days of ICU stay were sampled respiratory specimens. Incidence rate and distribution of pathogens were calculated and logistic regression analysis was performed.

Results: A total 1520 patients developed VAP (21\%). The most common microorganisms found were Staphylococcus aureus (43\%), Steptococcus pneumoniae (22\%) and Pseudomonas aeroginosa (18\%) and Klebsiella pneumoniae (8\%) and Haemophilus influenzae (6\%) and others (3\%). Multi-drug resistant (MDR) pathogens were found $43.3 \%$ of the VAP. Of the S. pneumoniae isolates, $31.4 \%$ were MDR and inappropriate initial antimicrobial treatment $(23 \%)$ were significantly higher. Patients with VAP had greater mortality rate and longer duration of ICU stay $(p<0.001)$. The mortality was $42 \%$ among VAP caused by S. pneumoniae.

Conclusion: VAP was the most common nosocomial infection in ICU. The incidence rate of S. pneumoniae is rapidly going up and MDR serotypes were growing with progressive way in ICU. VAP with S. pneumoniae was one of the leading causes of ICU mortality after Staphylococcus aureus. Increasing prevalence of MDR pathogens in early VAP indicate that inappropriate use of antibiotics are the leading cause of it. VAP was the main predicting factor for mortality and ICU stay with much greater costs of ICU.

No conflict of interest

ISPPD-0364

Pneumococcal Pneumonia---Risky Business

\section{BACTERIAL PNEUMONIA: AN OVERVIEW OF OCCURRENCE IN A TERTIARY CARE CENTRE IN INDIA}

S. Surapaneni ${ }^{1}$, S. Surapaneni ${ }^{1}$

${ }^{1}$ Pediatrics, Pragna Hospital, Hyderabad, India

Background and Aims: The data regarding age specific rates of disease and distribution of the organisms causing pneumonia in children is limited in India. Identification of the etiologic agent is particularly difficult in pediatric populations. The study is designed to study the etiology of community-acquired bacteremic pneumonia in children. Methods: This is a hospital based prospective study. Children from age group 2 months to 5 years with symptoms suggestive of cough, cold and fever (temperature $\geq 39^{\circ} \mathrm{C}$ ) of duration $>3$ days were enrolled. Children with history of any known significant congenital or acquired disease, or clinically diagnosed febrile illnesses were excluded. All the children were investigated by blood culture, C-reactive protein (CRP), complete blood count (CBC) and chest X-ray. Results: Majority $(115,35.49 \%)$ were of age group 13 months to 2 years followed by age group 2 months to 12 months $(56,17.28 \%) .57$ (17.59\%) patients showed blood culture positivity; majority (8.02\%) of them were in age group 13-24 months, whereas least (1.54\%) were in age group 37 months-48 months. Most commonly isolated bacteria were Staphylococcus aureus (31.57\%), Klebsiella pneumoniae (24.57\%), and Staphylococcus spp.(21.02\%). $239(73.76 \%)$ had history of exposure to a smoker in the family, out of whom $47(14.5 \%)$ were culture positive. Among the 85 patients not exposed to passive smoking, $10(3.08 \%)$ were blood culture positive.

Conclusion: The preliminary study results documents the common bacterial pathogens causing pneumonia in children and provides evidence to policy makers to strengthen the child health programs in India.

Conflict of interest 
ISPPD-0419

Pneumococcal Pneumonia---Risky Business

\section{VIRAL AND BACTERIAL COLONIZATION AMONG PNEUMONIA PATIENTS IN TONE DISTRICT, TOGO}

J.C. Moïsi ${ }^{1}$, J.N. Telles ${ }^{2}$, H. Tall ${ }^{3}$, B.M. Njanpop-Lafourcade ${ }^{4}$, A. Rajoharison ${ }^{2}$, K. Agbenoko ${ }^{5}$, M.S. Makawa ${ }^{6}$, M. Amidou $^{7}$, J.E. Mueller ${ }^{8}$, G. Paranhos-Baccalà' ${ }^{2}$, B.D. Gessner ${ }^{1}$, T.S. Tamekloe ${ }^{9}$

${ }^{1}$ Epidemiology, Agence de Médecine Préventive, Paris, France; ${ }^{2}$ Emerging Pathogens Laboratory, Fondation Mérieux, Lyon, France; ${ }^{3}$ Epidemiology, Agence de Médecine Préventive, Ouagadougou, Burkina Faso; ${ }^{4}$ Laboratory expertise, Agence de Médecine Préventive, Paris, France; ${ }^{5}$ Regional Hospital Laboratory, Ministry of Health Togo, Dapaong, Togo; ${ }^{6}$ Direction Provinciale de la Santé de Tône, Ministry of Health Togo, Dapaong, Togo; ${ }^{7}$ Direction Regionale de la Santé des Savanes, Ministry of Health Togo, Dapaong, Togo; ${ }^{8}$ Epidemiology, Ecole des Hautes Etudes en Santé Publique, Paris, France; ${ }^{9}$ Epidemiology Division, Ministry of Health Togo, Lomé, Togo

Background and Aims: We aimed to describe viral and bacterial colonization among pneumonia patients in Tone District, northern Togo.

Methods: From August 2011 to July 2012 we obtained blood for culture and real-time multiplex PCR (rtm-PCR) for bacterial identification, nasal aspirates for bacterial and viral testing by rtm-PCR respiratory 21plus (Fast Track Diagnostics)and chest radiographs for assessment of WHO endpoint pneumonia (lobar consolidation or pleural effusion) from patients admitted for clinical pneumonia at the five hospitals in Tone District. Pneumococcal serotyping was performed by Quellung and rtm-PCR.

Results: Among 342 nasal aspirates, $29 \%$ were positive for bacteria alone, $15 \%$ for viruses alone and $18 \%$ for both. The most common viruses were rhinovirus ( $n=34)$, influenza A B and H1N1 $(n=30)$, and hMPV ( $n=18)$; five patients had RSV. Pneumococcus was identified in 133 cases (39\%) Staphylococcus aureus in 41 (12\%) and Haemophilus influenzae type $b$ in 9 (3\%). Children $<5$ were more likely to be colonized than persons $5+$ years for bacteria $(65 \%$ vs. $44 \%, p<0.01 ; 56 \%$ vs. $36 \%$ pneumococcus, $p<0.01)$ and viruses $(58 \%$ vs. $28 \%, p<0.01)$. Pneumococcal carriage was associated with WHO endpoint pneumonia ( $47 \%$ vs. $25 \%$ in $5+, p<0.01 ; 63 \%$ vs. $48 \%$ in $<5, p=0.27$ ).Among 39 patients with blood culture or PCR positive for pneumococcus, 21 (53\%) were colonized of which 18 had concordant serotypes in blood and the nasopharynx.

Conclusion: Several viruses and bacteria were frequently identified among pneumonia patients. Data from healthy controls are needed to determine the role of each pathogen in the etiology of pneumonia.

Conflict of interest

ISPPD-0262

Pneumococcal Pneumonia---Risky Business

\section{PICTURE: CLINICAL PATTERN AND PNEUMOCOCCAL INFECTIONS IN PATIENTS WITH COMMUNITY-ACQUIRED PNEUMONIA (CAP) ADMITTED TO EMERGENCY DEPARTMENT}

F. Blasi ${ }^{1}$, P. Tarsia ${ }^{1}$, L.C. Morlacchi ${ }^{1}$, A. Gramegna ${ }^{1}$, G. Salomoni ${ }^{1}$, G. Spoletini ${ }^{1}$, C. Travierso ${ }^{1}$, V. Rossetti ${ }^{1}$, C. Azzari $^{2}$

${ }^{1}$ CARDIOPULMONARY, IRCCS Foundation Ca' Granda Ospedale Maggiore Policlinico, Milan, Italy; ${ }^{2}$ Health Science, Pediatric Section Department of Health Sciences University of Florence and Anna Meyer Children's University Hospital, Florence, Italy

PICTURE study is a single-centre observational cross-sectional and prospective study on clinical pattern and pneumococcal infections in patients with community-acquired pneumonia (CAP).

Primary objective: To describe the proportion of community-acquired pneumonia that is attributable to Streptococcus pneumoniae (SP) in hospitalized CAP patients.

Secondary objectives: To describe CAP attributable to SP according to socio-demographic and clinical variables; To describe clinical evolution of CAP; To define the proportion of bacteriaemic and non bacteriaemic episodes at hospital admission; To describe the serotype distribution and antibiotic resistance patterns of pneumococcal isolates; To estimate the in-hospital and 30-days after discharge mortality and hospital resource utilization. The serotype distribution and antibiotic resistance patterns of pneumococcal isolates (at admission or during hospitalization) have been evaluated. A total number of 193 patients, $59 \%$ males, mean $( \pm S D)$ age $72.9( \pm 15.8)$ y, were enrolled; 14 patients died during hospitalization; 4 patients died after discharge; 7 patients were untraceable after discharge and 1 withdrew inform consent (after discharge). A total number of 35 patients (18\%) had CAP due to SP. Thirty-one samples were positive to SP and had typeable serotype (table) (more than 1 serotype in each sample). In our series SP is the main cause of CAP. The 13-valent conjugate vaccine potentially covers more than $40 \%$ of isolated serotypes.

\begin{tabular}{|l|l|}
\hline Serotype & $\mathrm{n}(\%)$ \\
\hline $35 \mathrm{~F}$ & $9(16)$ \\
\hline 3 & $7(12)$ \\
\hline 24 & $5(9)$ \\
\hline $6,7 \mathrm{~F}, 18$ & $3(5)^{*}$ \\
\hline $23 \mathrm{~B}, 14,11,21,1,5$ & $2(3)^{*}$ \\
\hline $38,22,23 \mathrm{~F}, 19 \mathrm{~A}, 19 \mathrm{~F}, 2,12,8$ & $1(2)^{*}$ \\
\hline TOTAL & $57(100)$ \\
\hline *each serotype & \\
\hline
\end{tabular}


ISPPD-0521

Pneumococcal Pneumonia---Risky Business

\title{
FACTORS AFFECTING PNEUMOCOCCAL BLOOD CULTURE YIELD IN CHILDREN <5 YEARS WITH SEVERE OR VERY SEVERE PNEUMONIA IN THE PERCH STUDY
}

\author{
The PERCH Study Group ${ }^{1}$ \\ ${ }^{1}$ International Vaccine Access Center, The Johns Hopkins Bloomberg School of Public Health, Baltimore, USA
}

Background: Blood culture is the gold standard diagnostic test for pneumococcal pneumonia. Factors that potentially influence blood culture positivity were examined in the Pneumonia Etiology Research for Child Health (PERCH) study.

Methods: Participants were children $28 \mathrm{~d}-59$ mo in 7 African and Asian countries hospitalized with severe or very severe pneumonia. Blood cultures were processed on automated systems with bottles incubated for $\geq 5$ days, unless positive. Antibiotic pretreatment was assessed by serum bioassay and clinician report.

Results: Of 3360 specimens cultured to date, 139 (4.1\%) yielded a non-contaminant organism (range $0 \%-7.3 \%$ across sites); 40 (1.2\%) yielded Streptococcus pneumoniae. S. pneumoniae positivity (range 0-3.6\%), antibiotic pretreatment and blood culture volumes varied by site (Figure). Using multivariate logistic regression adjusting for site, blood culture positivity for $S$. pneumoniae was significantly associated with very severe pneumonia (OR $3.33 ; 95 \% \mathrm{Cl} 1.63,6.80$ ) and HIV infection (OR 9.51; 95\% $\mathrm{Cl} 3.77,24.00$ ). Specimen volume $\geq 3 \mathrm{~mL}$ showed a trend to association with isolating pneumococcus (OR 1.94; $95 \% \mathrm{Cl} 0.84,4.47$ ), while antibiotic pretreatment showed a negative trend to association with isolating pneumococcus (OR $0.41 ; 95 \% \mathrm{Cl} 0.15,1.12$ ).

Conclusion: Pneumococcal yield from blood culture varied considerably by PERCH site. Disease severity and HIVstatus were significantly associated with pneumococcal-specific blood culture yield in this study population, while there were indications of a non-significant trend to association for blood volume and antibiotic pretreatment.

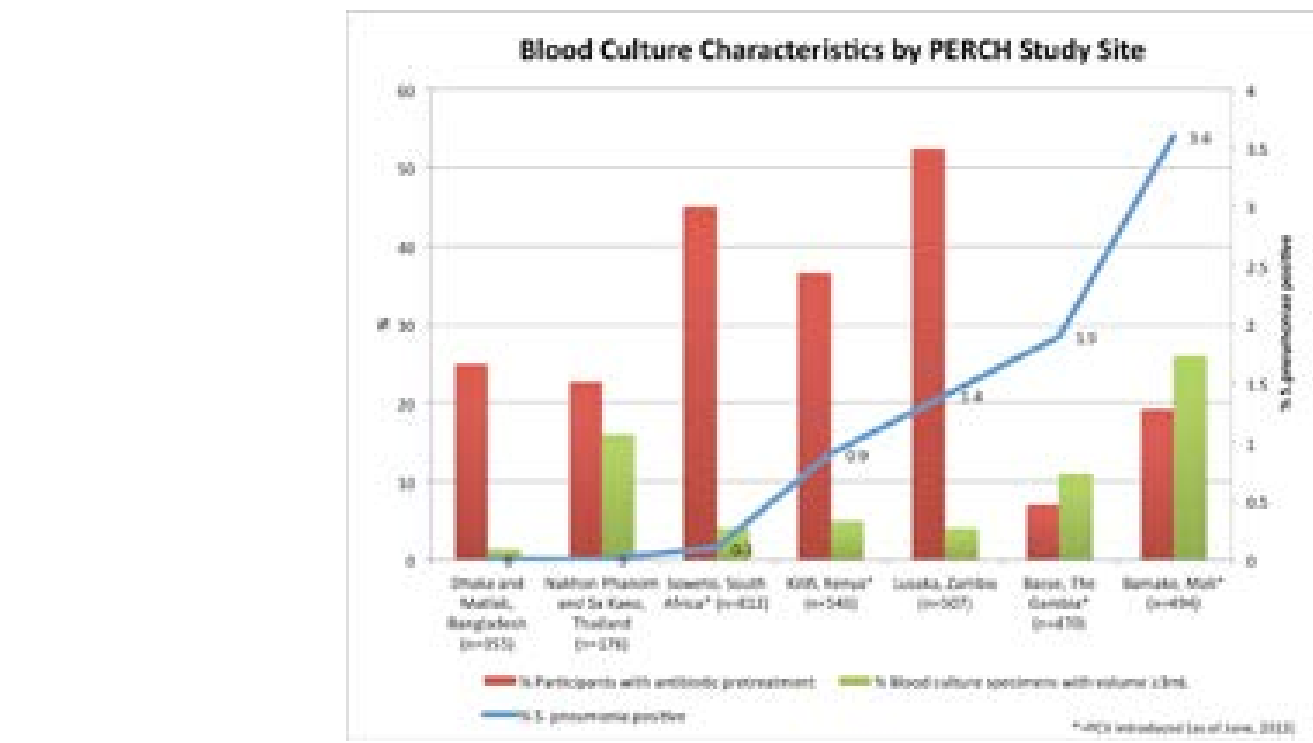

No conflict of interest

ISPPD-0263

Pneumococcal Pneumonia---Risky Business

\section{THE IMPACT OF 7-VALENT PNEUMOCOCCAL CONJUGATE VACCINE (PCV7) ON PNEUMONIA IN WESTERN EUROPE AND NORTH AMERICA: A LITERATURE REVIEW}

M. Tin Tin Htar ${ }^{1}$, P. Balmer ${ }^{1}$, M. Haugh ${ }^{1}$, H. Madhava², D. Christopoulou ${ }^{2}$, D. Menegas ${ }^{3}$, E. Bonnet ${ }^{1}$

${ }^{1}$ ESAT Vaccines, Pfizer International Operations, Paris, France; ${ }^{2}$ Vaccines, Pfizer Ltd, Walton on the Hill, United Kingdom; ${ }^{3}$ Vaccines, Pfizer Hellas $\mathrm{AE}$, Athens, Greece

Background and Aims: Prevenar (PCV7) was licensed for the prevention of pneumococcal diseases in 2000 and was replaced by PCV13 in 2009. This review aimed to evaluate the impact of PCV7 on pneumonia in Western Europe and North America.

Methods: A literature review was performed from 2000 to 2013 to assess the impact of PCV7 on pneumococcaland all-cause-pneumonia related hospitalization and healthcare visits.

Results: Twelve studies (Europe $n=4$; US $n=8$ ) reported the impact on all-cause pneumonia hospitalization and six also reported the impact on pneumococcal pneumonia hospitalization. In children $<2$ years, a reduction of 32 to $71 \%$ (median $57 \%$ ) and 15 to $52 \%$ (median 27\%) was observed for pneumococcal and all-cause pneumonia hospitalization, respectively. In those aged >65 years, a reduction of 38 to 55\% (median $42 \%$ ) was reported for pneumococcal pneumonia hospitalization whereas for all-cause pneumonia hospitalization the impact varied from a reduction of $23 \%$ to an increase of $44 \%$ (median reduction $7 \%$ ). In other age groups there was a median reduction of $40 \%$ (range: $16-63 \%$ ) and $7 \%$ (range: $+72 \%$ to $-56 \%$ ) for pneumococcal and all-cause pneumonia hospitalization, respectively. 
Conclusion: The reported impact varied by age, case definition (pneumococcal- or all-cause- pneumonia) and periods compared, particularly the time after PCV7 introduction and the definition of the pre-PCV7 period. There was a general reduction in pneumococcal pneumonia in all age groups; the reduction in all-cause-pneumonia was more clearly visible in children $<2$ years.

Conflict of interest

ISPPD-0294

Pneumococcal Pneumonia---Risky Business

\section{EFFECTIVENESS OF PNEUMOCOCCAL CONJUGATE VACCINE AGAINST PNEUMONIA IN ISRAEL: A MATCHED CASE- CONTROL ANALYSIS USING SURVEILLANCE DATA}

J.R. Verani ${ }^{1}$, N. Givon-Lavi ${ }^{2}$, C.G. Whitney ${ }^{1}$, D. Greenberg ${ }^{2}$, R. Dagan ${ }^{2}$

${ }^{1}$ Respiratory Diseases Branch, Centers for Disease Control and Prevention, Atlanta, USA; ${ }^{2}$ Pediatric Infectious Diseases Unit, Soroka University Medical Center and the Faculty of Health Sciences Ben-Gurion University, Beer-Sheva, Israel

Background: Pneumococcal conjugate vaccines (PCVs) are an important tool for reducing the burden of pediatric pneumonia. However, relatively little is known about their effectiveness against pneumonia in routine immunization programs. Israel introduced 7-valent PCV (PCV7) in July 2009 and transitioned to 13-valent (PCV13) in November 2010.

Methods: We utilized data from population-based, on-going surveillance for pediatric pneumonia and gastroenteritis at a large hospital in southern Israel for a case-control analysis of PCV effectiveness against pneumonia. Cases were defined as alveolar pneumonia on chest radiograph in children age-eligible for $\geq 1$ PCV dose. Controls were selected from children with rotavirus-negative gastroenteritis and individually matched to cases by ethnicity (Jewish/Bedouin), birth date (+/- 1month) and medical attention date (+/- 1 month). We estimated effectiveness as 1 - matched odds ratio for PCV vaccination.

Results: From July 2009 to June 2013, 2,446 cases were enrolled in surveillance; 738 (30.2\%) were matched to 1,688 controls (median, 2 controls per case; range 1-20). The median age of matched cases was 12.4 months (range 2-59), and 65.4\% were Bedouin. Overall, 578 (78.3\%) cases and 1370 (81.2\%) controls had received $\geq 1$ PCV (PCV7 or PCV13) dose; $49.5 \%$ of PCV doses with data on formulation were PCV13. The effectiveness of $\geq 2$ PCV doses was 40.6\% (95\% Cl: 11.1, 60.3\%).

Conclusion: PCV is protective against pneumonia in the routine immunization program in Israel. The results reflect a combined effectiveness of PCV7 and PCV13. Further analysis on the potential contribution of PCV7 vs. PCV13 is on-going, in an attempt to guide PCV13 policy decisions.

No conflict of interest

ISPPD-0343

Pneumococcal Pneumonia---Risky Business

\section{EPIDEMIOLOGY OF PNEUMOCOCCAL PNEUMONIA IN HOSPITALIZED ADULTS IN GUATEMALA}

C.L. Contreras ${ }^{1}$, J.R. Verani ${ }^{2}$, M.R. Lopez ${ }^{1}$, A. Paredes ${ }^{3}$, C. Bernart ${ }^{1}$, F. Moscoso ${ }^{1}$, W. Arvelo ${ }^{4}$, K.A. Lindblade ${ }^{4}$, J.P. McCracken $^{1}$

${ }^{1}$ Research Institute, Universidad del Valle de Guatemala, Guatemala City, Guatemala; ${ }^{2}$ National Center for Immunization and Respiratory Diseases, Centers for Disease Control and Prevention, Atlanta, USA; ${ }^{3}$ National Center of Epidemiology, Ministry of Health, Guatemala, Guatemala; ${ }^{4}$ Global Disease Detection Branch, Centers for Disease Control and Prevention, Atlanta, USA

Background: Streptococcus pneumoniae is a leading cause of pneumonia worldwide. However the burden of pneumococcal pneumonia among adults in low- and middle-income countries is not well described.

Methods: We analyzed data from 2008-2012 from two surveillance sites in Guatemala to describe pneumococcal pneumonia in adults. Cases were defined as a positive pneumococcal urinary antigen test or blood culture in persons $\geq 18$ years of age hospitalized with a sign/symptom of respiratory disease and evidence of acute infection. We calculated incidence rates using census data, and adjusted incidence accounting for missing testing results and healthcare utilization surveys in the surveillance areas.

Results: Among 1595 adults admitted with respiratory signs/symptoms and evidence of acute infection, 1315 (82\%) had either urine testing $(n=1007)$ or blood culture $(n=60)$ performed. We detected 182 cases of pneumococcal pneumonia; all positive by urine and six also by blood culture. The overall observed incidence of pneumococcal pneumonia was $8.1 / 100,000$ persons per year, with the lowest rate among $18-39$ year-olds $(3.1 / 100,000)$ and the highest among $\geq 65$ year olds $(36.5 / 100,000)$. The adjusted incidence of hospitalized pneumococcal pneumonia was $13 / 100,000$ overall and $58.4 / 100,000$ among $\geq 65$ year olds. There was no clear seasonality or trends over time in incidence. Overall in-hospital mortality was $5 \%$.

Conclusion: We show an important burden of hospitalized pneumococcal pneumonia in adults, particularly the elderly. However, even adjusted rates likely underestimate the true burden in the community. These data provide a useful baseline against which to measure the indirect effects of the recent introduction of the pneumococcal conjugate vaccine in Guatemala.

No conflict of interest 
ISPPD-0037

Pneumococcal Pneumonia---Risky Business

\title{
INDOOR AIR POLLUTION AND ITS EFFECT ON HUMAN HEALTH IN RURAL HILLY AREA OF DHANKUTA DISTRICT, NEPAL
}

\author{
P.K. Pokharel ${ }^{1}$, D. Tamrakar ${ }^{1}$, D.K. Yadav ${ }^{1}$ \\ ${ }^{1}$ Community Medicine, BP Koirala Institute of Health Science, Dharan, Nepal
}

Background and Aims: Approximately $76 \%$ of all global particulate matter air pollution occurs indoors in the developing world. High concentrations of indoor air pollutants lead to a number of adverse health consequences. Aim of the study was to find out the prevalence of the indoor air pollutio and its effect on human health especially among women and children.

Methods: Descriptive cross sectional study design was done in hilly area of Dhankuta district of Eastern Nepal. Data was collected with pretested semi-quantitative questionnaire by interview, observation checklist and physical clinical examination was done. Data was entered in excel programmed further analyzed into SPSS.

Results: A total of 168 houses were enrolled and interviewed systematically, majority(85\%) were Hindus, $67.9 \%$ were living in semi-pucca house and more than $41.7 \%$ were below poverty line. Majority (91.1\%) of people use biomass as kitchen fuel and $85 \%$ did not have exhaust outlet. Regarding time spend in kitchen $84.5 \%$ worked in kitchen 2-4 hours where as $7.1 \%$ more than $4 \mathrm{hrs}$. Health related problems that may be due to indoor air pollution in the women working in the kitchen, accordingly $38.7 \%$ was found to have some kind of breathlessness, $32.7 \%$ had cough and $23.2 \%$ produce phlegm. Out of total 13 were suffered from pneumonia, 6 suffered from bronchitis and 2 suffered of chronic obstructive pulmonary disease (COPD) while 7 were suffering from bronchial asthma. Conclusion: The study was revealed that $28 \%$ had severe Indoor air pollution. Around $29 \%$ had some kind of respiratory problem, in which pneumonia was common.

No conflict of interest

ISPPD-0537

Pneumococcal Pneumonia---Risky Business THE PATHOPHYSIOLOGICAL COMPARISON OF SECONDARY PNEUMOCOCCAL PNEUMONIA AFTER H1N1 PANDEMIC
2009 OR H1N1 NEW CALEDONIA INFLUENZA VIRUS INFECTION.

N. Yamamoto ${ }^{1}$, Y. Akeda ${ }^{2}$, S. Hamaguchi ${ }^{1}$, M. Seki ${ }^{1}$, K. Oishi $^{3}$, K. Tomono ${ }^{1}$

${ }^{1}$ Division of Infection Control and Prevention, Osaka University Graduate School of Medicine, Osaka-Suita, Japan; ${ }^{2}$ Laboratory of Clinical Research on Infectious Diseases, Research Institute for Microbial Diseases Osaka University, Osaka-Suita, Japan; ${ }^{3}$ Director, Infectious Disease Surveillance Center National Institute of Infectious Diseases, Osaka-Suita, Japan

Background: The outbreak of H1N1 pandemic 2009 influenza virus (PDM) caused high morbidity and mortality. The fatal cases were related with bacterial co-infection by Streptococcus pneumoniae (Sp), Staphylococcus pyogenes, and Staphylococcus aureus. There have been only a few studies reported about secondary pneumococcal pneumonia after PDM. In this study, we compared the secondary pneumococcal pneumonia in mice after PDM or H1N1 seanonal influenza virus (NC).

Method: Mice were infected intranasally with PDM or NC, followed 5 days later by nasal infection with Sp. At 6, 16, and 48 hour after the pneumococcal infection, lung and blood samples were obtained to examine on bacterial and viral titer, cytokines expression and lung histopathology.

Result: The bacterial density increased similarly in both PDM/Sp co-infected mice (PDM mice) and NC/Sp coinfected mice (NC mice). Compared with NC mice, PDM mice showed higher level of inflammatory cytokines, but lower level of antiinflammatory cytokines. In the histopathology of the lung tissues of PDM mice, NS1 antigens from H1N1 influenza virus were detected in the alveolar and bronchoalveolar epithelium. In contrast, they were found only in the bronchoalveolar epithelium of NC mice.

Conclusion: Our data indicated that PDM was more invasive in lungs and provoked severe inflammation in comparison with NC. In contrast, the bacterial load in the lungs after influenza infection was comparable. These results suggested that enhanced inflammation played a critical role to establish severe clinical manifestation of secondary bacterial infection after PDM infection.

No conflict of interest 
ISPPD-0005

Pneumococcal Pneumonia---Risky Business

\title{
EVALUATION OF THE RESULTS OF THE FIRST MASS VACCINATION AGAINST PNEUMOCOCCAL DISEASE IN THE REPUBLIC OF KAZAKHSTAN
}

\author{
L.T. Yeraliyeva' ${ }^{1}$, B.A. Ramazanova² ${ }^{2}$ K.K. Mustafina², N.Y. Azymbayeva ${ }^{3}$ \\ ${ }^{1}$ Children Infectious, S.D. Asfendiyarov Kazakh National Medical University, Almaty, Kazakhstan; ${ }^{2}$ Microbiology, S.D. Asfendiyarov Kazakh Nation- \\ al Medical University, Almaty, Kazakhstan; ${ }^{3}$ Epidemiology, Kazakh State Sanitarium Inspection of Ministry of Health of RK, Almaty, Kazakhstan
}

Pneumococcal disease is a serious medical and social problem in many countries of the world, including Kazakhstan. The only intelligent solution to the problem of pneumococcal carrier, decrease of a large number of various forms of pneumococcal disease and development of antibiotic resistance of pneumococcus - is vaccination. Since 2010, in 2 regions of Kazakhstan, a stepped vaccination against pneumococcal disease has been started and by 2015 these activities will be implemented throughout the country.

Aims: To evaluate the results of 3-year vaccination (2010-2012) against pneumococcal disease in 2 regions of Kazakhstan.

Materials and Methods: Analysis of statistic data on pneumococcal disease incidence and acute otitis media and infant mortality from pneumonia. (Statistic book "Health of the Republic of Kazakhstan and the activity of health care organizations in 2010, 2011, 2012").

Results: There is a decrease of the incidence of pneumonia per 1000 infants in the East Kazakhstan region by 46\%, and accordingly by $49.6 \%$ in the Mangystau region. Incidence of acute otitis media has also been decreased in the East Kazakhstan region by $21.3 \%$, and in the Mangystau region in 2.1 times from $19.8 \%$ to $9.3 \%$. Infant mortality from pneumonia (per 10,000 live-birth infants) in the East Kazakstan region has been decreased by $15.5 \%$ and in the Mangystau region by $57.1 \%$.

Conclusion: Implementation of specific vaccination against pneumococcal disease will reduce infant and child mortality and disease incidence, and may also have impact on the total mortality due to the producing effect of the community immunity.

No conflict of interest

ISPPD-0216

Pneumococcal Pneumonia---Risky Business

\author{
LOW SENSITIVITY OF URINARY ANTIGEN TESTING IN PATIENTS WITH PNEUMOCOCCAL PNEUMONIA \\ C. Yoo ${ }^{1}, \mathrm{H} . \mathrm{KoO}^{1}$, C. Lee ${ }^{1}$ \\ ${ }^{1}$ Internal Medicine, Seoul National University College of Medicine, Seoul, Korea
}

Purpose: The aim was to evaluate the sensitivity of the urinary pneumococcal antigen test (UPA) for diagnosing pneumococcal pneumonia in a clinical setting and verify the factors associated with false-negative UPA results. Methods: Between January 2005 and December 2007, adult patients admitted with pneumococcal pneumonia were enrolled retrospectively and the severity of community-acquired pneumonia CAP was assessed by CURB-65 score.

Results: A total of 180 patients were included in the study. One hundred sixty patients (88.9\%) had Streptococcus pneumoniae in respiratory specimens, 20 patients $(11.1 \%)$ in blood, and one patient $(0.6 \%)$ in pleural fluid. Eightyfour patients were UPA positive and the sensitivity of the test was $46.7 \%$ ( $95 \% \mathrm{Cl}, 0.37-0.56)$. The UPA had an $80 \%$ sensitivity in patients with pneumococcal bacteremia, whereas the sensitivity was $39.4 \%$ in patients without bacteremia. In a multivariate analysis, a false-negative result on the UPA was associated with a lower C-reactive protein (CRP) level $(\leq 10 \mathrm{mg} / \mathrm{L})$, lower CURB65 score $(\leq 2)$, older age, underlying lung disease and absence of cerebrovascular accident.

Conclusion: UPA showed a comparatively low sensitivity in microbiologically proven CAP. Older age, underlying lung disease, absence of cerebrovascular accident, lower CRP level and lower CURB-65 score, were independently associated with a false-negative result on the UPA.

No conflict of interest 
ISPPD-0131

Pneumococcal Pneumonia---Risky Business

\section{ETIOLOGY AND CLINICAL CHARACTERISTICS OF <5 YEARS HOSPITALIZED CHILDREN WITH SEVERE ACUTE RESPIRATORY INFECTION IN SUZHOU, CHINA}

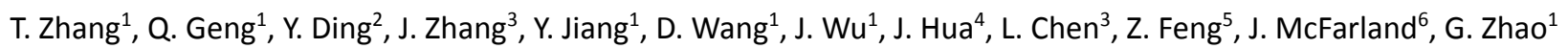

${ }^{1}$ Epidemiology, School of Public Health Fudan University, Shanghai, China; ${ }^{2}$ Laboratory, Soochow University Affiliated Children Hospital, Suzhou, China; ${ }^{3}$ Infectious Diseases, Suzhou Center for Disease Control and Prevention, Suzhou, China; ${ }^{4}$ ICU, Soochow University Affiliated Children Hospital, Suzhou, China; ${ }^{5}$ EID, Chinese Center for Disease Control and Prevention, Beijing, China; ${ }^{6}$ China office, US Center for Disease Control and Prevention, Beijing, China

Background and Aims: Severe acute respiratory infection (SARI) is the leading cause of hospitalization in children. This study was to learn the etiologies and the clinical characteristics of SARI in Chinese children.

Methods: Since March 2011, a surveillance study on all SARI children was conducted in Soochow University Affiliated Children's Hospital. Nasopharyngeal aspirate, blood or pleural effusion bacterial culture, real-time PCR (rtPCR) or direct immunofluorescent antigen test for virus were used to identify the possible pathogens.

Results: From March 2011 to June 2013, a total of 2164 children $<5$ years were included and $61.2 \%$ of them were male. The mean age was $1.73 \pm 1.36$ years. There were $1845(85.3 \%)$ children with proven pneumonia. The combined tests identified at least one pathogen in $62.2 \%$ of all cases. The most common pathogens were Streptococcus pneumoniae (Sp,17.5\%), respiratory syncytial virus (RSV,14.6\%), influenza virus $(12.5 \%)$ and Haemophilus influenzae $(11.3 \%)$. The median length of hospital stay was 7 days. For the treatments during hospitalization, $98.2 \%$ of them received antibiotics, $81.3 \%$ received anti-viral agents, $76.0 \%$ had glucocorticoids, $37.7 \%$ required oxygen treatment and $2.0 \%$ was referred to ICU. The mean cost for hospitalization was 4898.8RMB (around US\$777.6). Children with RSV infection were significantly younger than those with influenza $(p<0.05)$ or Sp infection $(p<0.05)$. Compared with non-Sp infection children, the Sp infection children had significantly higher hospitalization cost $(p<0.05)$ and longer hospital stay $(p<0.05)$.

Conclusion: Sp, RSV and influenza were the common etiologic agents of childhood SARI in Suzhou, China. Our findings underscore the high burden of hospitalization for SARI in young children.

No conflict of interest 